\title{
Reduced-order modelling of radiative transfer effects on Rayleigh-Bénard convection in a cubic cell
}

\author{
Laurent Soucasse $^{1} \dagger$, Bérengère Podvin ${ }^{2}$, Philippe Rivière $^{1}$ and \\ Anouar Soufiani ${ }^{1}$ \\ ${ }^{1}$ Laboratoire EM2C, CNRS, CentraleSupélec, Université Paris-Saclay, 8-10 rue Joliot Curie, \\ 91192 Gif-sur-Yvette, France \\ ${ }^{2}$ LIMSI, CNRS, Université Paris-Saclay, Bât 507, rue du Belvédère, Campus Universitaire, \\ 91405 Orsay, France
}

(Received xx; revised xx; accepted $\mathrm{xx}$ )

This paper presents a reduced-order modelling strategy for Rayleigh-Bénard convection of a radiating gas, based on the proper orthogonal decomposition (POD). Direct numerical simulation (DNS) of coupled natural convection and radiative transfer in a cubic Rayleigh-Bénard cell is performed for an air $/ \mathrm{H}_{2} \mathrm{O} / \mathrm{CO}_{2}$ mixture at room temperature and at a Rayleigh number of $10^{7}$. It is shown that radiative transfer between the isothermal walls and the gas triggers a convection growth outside the boundary-layers. Mean and turbulent kinetic energy increase with radiation, as well as temperature fluctuations to a lesser extent. As in the uncoupled case, the large-scale circulation (LSC) settles in one of the two diagonal planes of the cube with a clockwise or anticlockwise motion, and experiences occasional brief reorientations which are rotations of $\pi / 2$ of the LSC in the horizontal plane. A POD analysis is conducted and reveals that the dominant POD eigenfunctions are preserved with radiation while POD eigenvalues are increased. Two POD-based reduced-order models including radiative transfer effects are then derived: the first one is based on coupled DNS data while the second one is an a priori model based on uncoupled DNS data. Owing to the weak temperature differences, radiation effects on mode amplitudes are linear in the models. Both models capture the increase in energy with radiation and are able to reproduce the low-frequency dynamics of reorientations and the high-frequency dynamics associated with the LSC velocity observed in the coupled DNS.

Key words: low-dimensional models, Bénard convection

\section{Introduction}

It has been soon recognised that radiative transfer can significantly alter thermally driven flows encountered in atmospheric physics, in astrophysics or in various engineering applications. The emission and absorption of radiation affect the temperature of a radiating fluid, which, in turn control the buoyant motion. Water vapour and carbon dioxide are the most common radiating gases in the infrared and are present in significant quantity in the atmosphere or in confined environments such as buildings. First studies dedicated

$\dagger$ Email address for correspondence: laurent.soucasse@centralesupelec.fr 
to radiative transfer effects on natural convection have been focused on the onset of Rayleigh-Bénard convection (RBC), where a radiating fluid layer is confined between two horizontal plates, heated from the bottom and cooled from above. Using linear stability analysis, Goody (1956), followed by Spiegel (1960), have shown that radiative transfer delays the onset of convection and stabilizes the fluid layer. Two stabilizing physical mechanisms were highlighted: the decrease of the static temperature gradient in the core of the layer and the damping of thermal perturbations with radiative transfer. Though these studies were restricted to a grey fluid (radiative properties independent of the wavelength), the stabilizing effect of radiation has been further confirmed for real molecular gases (Bdéoui \& Soufiani 1997) and supported by experiments conducted with ammonia (Gille \& Goody 1964) or carbon dioxide (Hutchinson \& Richards 1999). The study of the stability of a radiating fluid layer exposed to a cold radiative background, which model the atmospheric nocturnal boundary-layer, also shows a delay of the onset of convection when radiative transfer is taken into account (Prasanna \& Venkateshan 2014).

While a substantial research effort has been devoted to understand turbulent RBC and predict the scaling laws for the Nusselt number (Grossmann \& Lohse 2000), the largescale organisation of the flow (Brown et al. 2005; Mishra et al. 2011), the turbulence properties (Lohse \& Xia 2010) or the plume dynamics (van der Poel et al. 2015), the study of radiative transfer effects on $\mathrm{RBC}$ at high Rayleigh numbers $R a$ has received little attention. The numerical investigation of radiative transfer effects is actually restricted by the computational time required for solving the radiative transfer equation in a turbulent participating medium. The radiative intensity $I_{\nu}(\boldsymbol{\Omega}, \boldsymbol{r}, t)$ varies in a seven-dimensional space (wavenumber $\nu$, propagation direction $\boldsymbol{\Omega}$, space $\boldsymbol{r}$ and time $t$ ): its discretisation needs to cover all the spatial and temporal scales of the turbulence but also all the propagation directions of the angular domain and all the wavenumbers of the spectrum. Experimental investigations are also challenging as non-intrusive measurement techniques are required when radiation comes into play and as radiating gases are associated with low thermal conductivities, making harder the insulation of the experimental devices.

Some authors have nevertheless attempted to account for radiative transfer in steady or weakly turbulent RBC. Lan et al. (2003) explored flow regimes above the onset of convection $\left(R a \sim 10^{3}\right)$ using three-dimensional (3D) coupled calculations for a grey radiating fluid. Mishra et al. (2014) also performed two-dimensional (2D) coupled calculations for a grey participating medium for Rayleigh numbers around $10^{4}$ and reported an increase of the number of convective cells with radiation. Radiation effects on the shape and on the number of large-scale convection rolls have also been observed by Sakurai et al. (2012) for mixed convection $\left(R a \sim 10^{6}\right)$ using an optically thin model for radiation. In weakly turbulent regime $\left(10^{6} \leqslant R a \leqslant 10^{7}\right)$, the coupled numerical results obtained by Soucasse et al. (2014a) for a real radiating gas in a cubic cell show that radiative transfer significantly increases the kinetic energy of the mean and fluctuating flows, though the results were obtained within a limited integration time. This convection intensification with radiation is also noticed in experiments where a lightspot serves as heat source for an absorbing fluid (Lepot et al. 2018; Bouillaut et al. 2019). Radiation is found to promote the mixing-length scaling regime as energy transfer is no longer restricted by the boundary-layers and can influence the flow field in the core of the cavity. The convection intensification with radiation in weakly turbulent regime seems to contradict the stabilizing effect described at low Rayleigh numbers. Interestingly, the same observations have been reported in differentially heated cavities where radiation is found to promote the turbulence in unsteady regime (Soucasse et al. 2016; Kogawa et al. 2017), while the onset of convection is delayed (Borget et al. 2001). It should be finally 
mentioned that surface-to-surface radiation can also affect $\mathrm{RBC}$ as shown for instance by Czarnota \& Wagner (2016) who consider radiative and conductive horizontal plates.

This brief literature review reveals a lack of reference results to understand the radiation effects on $\mathrm{RBC}$ but also a need of simple model able to capture these effects. Among the different modelling strategies for natural convection flows, reduced-order models based on proper orthogonal decomposition (POD) are established techniques that can capture the dynamics of turbulent large-scale flow structures. POD is a modal decomposition method that extracts a basis of orthogonal spatial modes from a statistical analysis of sampled flowfield data. POD-based low-order models are then derived using Galerkin projection of the Navier-Stokes equations onto a reduced set of POD modes, selected from an energy criterion. Although these models are mainly used to analyse numerical or experimental data a posteriori in a given configuration, they have been able to predict flow modifications due to a change in the flow parameters, such as variations of the turbulence intensity due to additional rates of strain (Lumley \& Podvin 1996). POD has been extensively used to analyse and model natural convection flows (Park et al. 2004; Verdoold et al. 2009; Bailon-Cuba et al. 2010; Podvin \& Sergent 2017).

The goal of this paper is to extend such kind of low-order model to account for radiative transfer. We have carried out a long term direct numerical simulation (DNS) of $\mathrm{RBC}$ at $R a=10^{7}$ in a cubic cell, for a radiating air $/ \mathrm{H}_{2} \mathrm{O} / \mathrm{CO}_{2}$ mixture at room temperature whose composition is relevant for building applications. Radiative transfer effects in these conditions have been analysed by comparison with uncoupled DNS results discussed in a previous study (Soucasse et al. 2019). Two POD-based reduced-order models including radiative transfer have been derived: the first one is based on coupled DNS data while the second one is an a priori model based on uncoupled DNS data. The linearisation of radiation emission (low temperature gradient assumption) is used to decompose the radiative source term into a sum of modal contributions associated with each POD temperature eigenfunction. The radiative contribution in the low-order models is then obtained by projecting this decomposition onto the POD basis. The problem configuration as well as the numerical methods used to perform the coupled DNS are given in section 2. Radiative transfer effects are analysed from numerical results in section 3. Finally, model derivation and model results are discussed in section 4 .

\section{Direct numerical simulations of coupled natural convection and radiation}

\subsection{Problem setup and governing equations}

We consider a cubical cavity of size $L$, heated from below and cooled from above. The cavity is filled with a radiating air $/ \mathrm{H}_{2} \mathrm{O} / \mathrm{CO}_{2}$ mixture, of molar composition $X_{\mathrm{H}_{2} \mathrm{O}}=0.02$ and $X_{\mathrm{CO}_{2}}=0.001$, at atmospheric pressure and at a mean temperature $T_{0}=300 \mathrm{~K}$. Top and bottom walls are maintained at uniform temperature $T_{\text {cold }}$ and $T_{\text {hot }}$ and the four lateral walls are assumed to be adiabatic. The six walls are characterised by uniform grey emissivities $\varepsilon$, the horizontal isothermal walls being black $(\varepsilon=1)$, while the vertical adiabatic walls being perfectly diffuse reflecting $(\varepsilon=0)$. The problem setup is displayed in figure 1. The Rayleigh number which controls the flow regime is set to $10^{7}$ and is given by $R a=g \beta \Delta T L^{3} /\left(\nu_{f} a\right)$, where $g$ is the gravitational acceleration, $\beta=1 / T_{0}$ is the thermal expansion coefficient, $\nu_{f}$ is the kinematic viscosity, $a$ is the thermal diffusivity and $\Delta T=\left(T_{\text {hot }}-T_{\text {cold }}\right)$ the temperature difference between hot and cold walls. The Prandtl number is set to 0.707 and is given by $\operatorname{Pr}=\nu_{f} / a$. Since radiation is treated in dimensional form, we also need to specify the size of the cavity, which is set to $L=1 \mathrm{~m}$. 
(a)

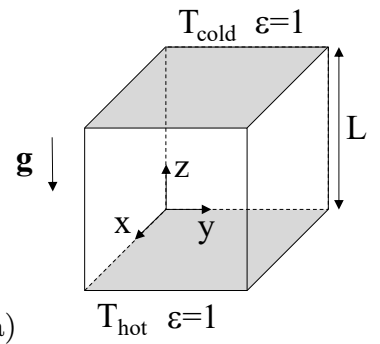

- side vertical walls adiabatic and reflecting $\varepsilon=0$

- filled with air- $\mathrm{H}_{2} \mathrm{O}-\mathrm{CO}_{2}$ mixture

(b)

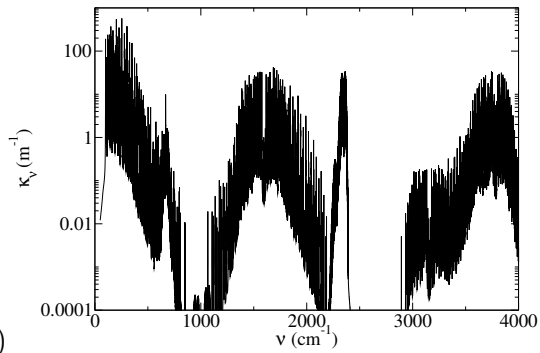

Figure 1. (a) Cubic Rayleigh-Bénard cell filled with a radiating air $/ \mathrm{H}_{2} \mathrm{O} / \mathrm{CO}_{2}$ mixture. Top and bottom walls are isothermal and black while side vertical walls are adiabatic and perfectly diffuse reflecting. (b) Absorption coefficient spectrum of the considered air $/ \mathrm{H}_{2} \mathrm{O} / \mathrm{CO}_{2}$ mixture (atmospheric pressure, $T_{0}=300 \mathrm{~K}, X_{\mathrm{H}_{2} \mathrm{O}}=0.02, X_{\mathrm{CO}_{2}}=0.001$ )

Governing equations under Boussinesq approximation are

$$
\begin{gathered}
\boldsymbol{\nabla} \cdot \boldsymbol{u}=0, \\
\frac{\partial \boldsymbol{u}}{\partial t}+\boldsymbol{u} \cdot \boldsymbol{\nabla u}=-\nabla p+\operatorname{Pr} \theta \boldsymbol{e}_{\boldsymbol{z}}+\frac{\operatorname{Pr}}{\sqrt{R a}} \nabla^{2} \boldsymbol{u}, \\
\frac{\partial \theta}{\partial t}+\boldsymbol{u} \cdot \boldsymbol{\nabla} \theta=\frac{1}{\sqrt{R a}}\left(\boldsymbol{\nabla}^{2} \theta+\mathcal{P}^{\mathrm{rad}}\right),
\end{gathered}
$$

where $\boldsymbol{u}=(u, v, w)$ is the dimensionless velocity vector, $p$ is the dimensionless motion pressure, $\theta$ is the reduced temperature and $\mathcal{P}^{\mathrm{rad}}$ is the dimensionless radiative power. A no-slip velocity condition $(\boldsymbol{u}=0)$ is prescribed on the six walls of the cavity and thermal boundary conditions are written as follows

$$
\left.\begin{array}{l}
\theta=0.5 \quad \text { on } \quad z=0 \\
\theta=-0.5 \quad \text { on } \quad z=1 \\
\boldsymbol{\nabla} \theta \cdot \boldsymbol{n}=0 \quad \text { on } \quad x=0, x=1, y=0, y=1
\end{array}\right\}
$$

where $x, y$ and $z$ are the dimensionless Cartesian coordinates. Note that there is no radiative flux on reflecting adiabatic side walls. Equations (2.1)-(2.3) are made dimensionless using the length of the cavity $L$, the reference time $L^{2} /(a \sqrt{R a})$ and the reduced temperature $\theta=\left(T-T_{0}\right) / \Delta T, T_{0}$ being the mean temperature between hot and cold walls.

The dimensionless radiative power is given by

$$
\mathcal{P}^{\mathrm{rad}}(\boldsymbol{r})=\frac{L^{2}}{\lambda \Delta T} \int_{\nu} \kappa_{\nu}\left(\int_{4 \pi} I_{\nu}(\boldsymbol{\Omega}, \boldsymbol{r}) d \boldsymbol{\Omega}-4 \pi I_{\nu}^{\circ}(T(\boldsymbol{r}))\right) d \nu,
$$

where $I_{\nu}(\boldsymbol{\Omega}, \boldsymbol{r})$ is the actual radiative intensity at wavenumber $\nu$, direction $\boldsymbol{\Omega}$ and position $\boldsymbol{r}, I_{\nu}^{\circ}(T(\boldsymbol{r}))$ is the Planck equilibrium intensity at temperature $T, \kappa_{\nu}$ is the absorption coefficient that is assumed to be uniform owing to the weak temperature differences and $\lambda$ is the thermal conductivity. The line-by-line (high resolution) absorption spectrum of the considered mixture is shown in figure 1. The shape of the spectrum makes the numerical evaluation of the integration over the wavenumbers in equation (2.5) computationally expensive. In order to save computational time, the ADF model (Pierrot et al. 1999) is used: it consists in substituting the integration over the wavenumber with an integration over the values $k$ of the absorption coefficient, for which a coarse logarithmic discretisation is sufficient. In the present study, the values of the absorption coefficient 
of figure 1 have been logarithmically discretised in $16 k$-classes. With the ADF-model, the radiative power writes

$$
\mathcal{P}^{\mathrm{rad}}(\boldsymbol{r})=\frac{L^{2}}{\lambda \Delta T} \sum_{j} k_{j}\left(\int_{4 \pi} I_{j}(\boldsymbol{\Omega}, \boldsymbol{r}) d \boldsymbol{\Omega}-4 w_{j} \sigma T^{4}(\boldsymbol{r})\right),
$$

where $k_{j}$ and $w_{j}$ are respectively the absorption coefficient and the weight associated with the $j^{\text {th }} k$-class and $\sigma$ is the Stefan-Boltzmann constant. $I_{j}(\boldsymbol{\Omega}, \boldsymbol{r})$ is the intensity field associated with the $j^{\text {th }} k$-class and satisfies the radiative transfer equation

$$
\boldsymbol{\Omega} \cdot \boldsymbol{\nabla} I_{j}(\boldsymbol{\Omega}, \boldsymbol{r})=k_{j}\left(\frac{w_{j} \sigma}{\pi} T^{4}(\boldsymbol{r})-I_{j}(\boldsymbol{\Omega}, \boldsymbol{r})\right),
$$

and boundary condition

$$
\left.I_{j}\left(\boldsymbol{\Omega}, \boldsymbol{r}^{w}\right)=\varepsilon \frac{w_{j} \sigma}{\pi} T^{4}\left(\boldsymbol{r}^{w}\right)\right)+\frac{1-\varepsilon}{\pi} \int_{\boldsymbol{\Omega}^{\prime} \cdot \boldsymbol{n}<0} I_{j}\left(\boldsymbol{\Omega}^{\prime}, \boldsymbol{r}^{w}\right)\left|\boldsymbol{\Omega}^{\prime} \cdot \boldsymbol{n}\right| d \boldsymbol{\Omega}^{\prime},
$$

for propagation direction $\boldsymbol{\Omega} \cdot \boldsymbol{n}>0, \boldsymbol{n}$ being the normal at boundary point $\boldsymbol{r}^{w}$ directed towards the inside of the domain. The accuracy of the present ADF-model has been shown to be better than $1 \%$ (Soucasse et al. 2012). Model parameters $\left(k_{j}, w_{j}\right)$ and details on the implementation can be found in Soucasse (2013).

It is worth noting that the flow equations are written and solved in dimensionless form, while radiative transfer equations are treated in dimensional form since we consider an actual molecular radiating gas. A key parameter for radiation is the optical thickness $\tau=k L$ that varies over several orders of magnitude in our model. Considering a grey fluid (wavelength-independent absorption, single $k$ value) would facilitate a parametric study of radiation effects but would fail to represent the behaviour of actual radiating gases.

Without radiation coupling, the problem satisfies four independent reflection symmetries $S_{x}, S_{y}, S_{z}$ and $S_{d}$ with respect to the planes $x=0.5, y=0.5, z=0.5$ and $x=y$ (Puigjaner et al. 2008). These four elementary symmetries generate a symmetry group of sixteen elements. In unsteady regime, we expect these symmetries to be satisfied by the time-averaged flow field. Radiation emission being proportional to $T^{4}$, radiative transfer should break the $S_{z}$ symmetry as the mean temperature gradient is directed along the $z$ axis. However, owing to the weak temperature gradients $(\Delta T \simeq 0.1 \mathrm{~K}$ for the conditions specified), non linear effects are negligible (namely $1-\frac{4 T_{0}^{3} \Delta T}{T_{\text {hot }}^{4}-T_{\text {cold }}^{4}} \simeq 3 \times 10^{-8}$ ) so that we can consider that the $S_{z}$ symmetry is still satisfied with radiation.

\subsection{Numerical methods}

\subsubsection{Flow field}

Equations (2.1)-(2.3) are solved using a Chebyshev collocation method for the three dimensions of space (Xin \& Le Quéré 2002). The pressure-flow coupling is ensured by a projection method that force the velocity divergence free condition. Time integration is performed through a second order temporal scheme combining a Backward Differentiation (BDF2) scheme for the linear terms with an Adams Bashforth extrapolation of convective terms. This algorithm is implemented for parallel computations applying domain decomposition along the $z$-vertical direction (Xin et al. 2008).

The spatial flow mesh is made of 81 Chebyshev collocation points in the $x$ and $y$ directions and $4 \times 21$ Chebyshev collocation points in the $z$ direction ( 4 spatial domains are constructed for parallelisation purposes, each discretized with 21 points). The number of spatial collocation points in the kinetic and thermal boundary-layers $\left(N_{u, B L}\right.$ and $\left.N_{\theta, B L}\right)$ 
is about 10 in the $x$ and $y$ directions and about 6 in the $z$ direction, which is satisfactory regarding the criterion proposed by Shishkina et al. (2010) $\left(N_{u, B L}=0.31 R a^{0.15}\right.$ and $N_{\theta, B L}=0.35 R a^{0.15}$ for $\left.P r \simeq 0.7\right)$. The thicknesses of the kinetic and thermal boundarylayers have been estimated using the correlations provided by the same authors and are respectively equal to $\delta_{u} / L=0.027$ and $\delta_{\theta} / L=0.031$ at $R a=10^{7}$. It will be shown in section 3 that radiation does not affect much the thermal and mechanical boundary-layer thicknesses in the case studied. The dimensionless flow time step is set to $2.5 \times 10^{-3}$.

\subsubsection{Radiation source term}

In order to determine the intensity field $I_{j}(\boldsymbol{\Omega}, \boldsymbol{r})$ and calculate the radiative power (2.6) that goes into the energy balance (2.3), a ray-tracing algorithm has been implemented. This approach consists in discretizing uniformly the $4 \pi$ solid angle domain into a large number of rays $N_{\Omega}$ and approximating angular integrals using

$$
\int_{4 \pi} I_{j}(\boldsymbol{\Omega}, \boldsymbol{r}) d \boldsymbol{\Omega}=\frac{4 \pi}{N_{\Omega}} \sum_{i=1}^{N_{\Omega}} I_{j}\left(\boldsymbol{\Omega}_{i}, \boldsymbol{r}\right) .
$$

The intensity $I_{j}\left(\boldsymbol{\Omega}_{i}, \boldsymbol{r}\right)$ is obtained by solving equation (2.7) along the ray of direction $\boldsymbol{\Omega}_{i}$ ranging from the current point $\boldsymbol{r}$ of abscissa $s=0$ to the boundary point $\boldsymbol{r}^{w}$ of abscissa $s=l$

$$
I_{j}\left(\boldsymbol{\Omega}_{i}, \boldsymbol{r}\right)=\int_{0}^{l} k_{j} w_{j} \frac{\sigma}{\pi} T^{4}(s) \exp \left(-k_{j} s\right) d s+I_{j}\left(\boldsymbol{\Omega}_{i}, \boldsymbol{r}^{w}\right) \exp \left(-k_{j} l\right) .
$$

The discretization of the spatial integration along the ray in the equation above is easily achieved using exact intersection calculations between the ray and the Cartesian mesh on which the temperature field is provided. The intensity field at boundary points, which is not known because of multiple reflections, is determined iteratively in a first step before computing the intensity field in the medium at points $\boldsymbol{r}$. The ray tracing algorithm is implemented in parallel by distributing the $N_{\Omega}$ rays among the different processors. This method has been validated against the Monte Carlo method and used in coupled calculations of natural convection and radiation (Soucasse et al. 2012, 2014b, 2016).

The spatial radiation mesh is made of 41 points in each spatial coordinate, obtained by coarsening the spatial flow mesh by a factor of two in each direction. We have checked that the use of this coarser grid is sufficient to capture all the spatial structures of the radiation field. Interpolations are used to get the temperature field on the radiation mesh and the radiation source term on the flow mesh. The angular mesh is made of $N_{\Omega}=900$ rays $\left(N_{\Omega}^{w}=450\right.$ rays from boundary points) which yields very good accuracy. The calculation is carried out for the $16 k$-classes of the ADF-model.

An explicit coupling is carried out between flow and radiation calculations. In practice it is sufficient to update the radiation field every 10 flow time steps: the flow time step is imposed by numerical stability constraints and does not correspond to significant variations of the temperature field, so that we can consider the radiation field constant over this period. Integration of Navier-Stokes equations and radiation equations are carried out simultaneously in an asynchronous way. Starting from a fluid at $\boldsymbol{u}=\mathbf{0}$ and $\theta=0$, coupled calculations are first carried out over a dimensionless time period of 2,400 in order to reach the asymptotic regime and then continued over a dimensionless time period of 10,000 to conduct the analysis. The total CPU cost of the coupled simulation is about 125,000 h (Intel Sandy Bridge E5-4650, $2.7 \mathrm{GHz}$ processors), $97 \%$ of this cost being spent for radiation computation. In the next section, coupled DNS results will be compared to uncoupled DNS results, where the gas is considered transparent $\left(\mathcal{P}^{\mathrm{rad}}=0\right)$. 


\section{Reduced-order modelling of radiative transfer effects on Rayleigh-Bénard convection 7}

Uncoupled results have been integrated over the same time period in the asymptotic regime and have been discussed in a previous study (Soucasse et al. 2019).

\section{Radiative transfer effects}

Radiative transfer effects are analysed in this section by comparing coupled DNS results to uncoupled DNS results. The effects on mean and fluctuating fields are discussed in section 3.1 and the effects on reorientations are discussed in section 3.2. Then, POD analysis of the coupled DNS is performed in section 3.3.

\subsection{Mean and fluctuating fields}

Figures 2 compares results obtained with and without radiation coupling for timeaveraged and fluctuating fields: the mean temperature $\langle\theta\rangle$, the mean kinetic energy $0.5\langle\boldsymbol{u}\rangle \cdot\langle\boldsymbol{u}\rangle$, the mean radiative power $\left\langle\mathcal{P}^{\mathrm{rad}}\right\rangle$, the half of temperature variance $0.5\left\langle\theta^{\prime} \theta^{\prime}\right\rangle$ and the turbulent kinetic energy $0.5\left\langle\boldsymbol{u}^{\prime} \cdot \boldsymbol{u}^{\prime}\right\rangle$, where $\langle\cdot\rangle$ denotes the time-average and ' denotes time fluctuations. These quantities are averaged over horizontal planes and plotted along the vertical direction. While mean and fluctuating temperature fields are not much affected by radiation coupling, there is a significant increase of kinetic energy of both mean and fluctuating velocity fields when coupling with radiation. The total kinetic energy increase (mean and fluctuating) is about $30 \%$. The mean radiative power plot shows that the hot (respectively cold) gas in the lower (resp. upper) half of the cavity is absorbing (resp. emitting), except in very thin emitting (resp. absorbing) layer near the hot bottom (resp. cold top) wall. The temperature in the cavity is thus slightly higher in the lower half of the cavity (slightly lower in the upper half) allowing a reinforcement of the velocity field that leads to an increase of velocity and temperature turbulent fluctuations. The increase of temperature fluctuations is however smaller because radiative transfer tends to damp temperature fluctuations (Soufiani 1991).

A quantity of interest in Rayleigh-Bénard convection is the total energy flux $q_{\text {tot }}$ in the vertical direction which is the sum of the conductive $q_{\text {cond }}$, convective $q_{\text {conv }}$ and radiative $q_{\text {rad }}$ energy fluxes

$$
q_{\mathrm{tot}}=\underbrace{-\frac{\partial\langle\theta\rangle}{\partial z}}_{q_{\mathrm{cond}}}+\underbrace{\sqrt{R a}\langle\theta w\rangle}_{q_{\mathrm{conv}}}+\underbrace{\frac{L}{\lambda \Delta T}\left\langle\sum_{j} \int_{4 \pi} I_{j} \boldsymbol{\Omega} \cdot \boldsymbol{e}_{\boldsymbol{z}} d \boldsymbol{\Omega}\right\rangle}_{q_{\mathrm{rad}}} .
$$

Averaged in the horizontal plane, this quantity is constant along $z$ because the side vertical walls are adiabatic. Figure 3 shows the three components of the total energy flux averaged in time and in the horizontal plane. Flux values at the wall $(z=0, z=1)$ and in the core $(z=0.5)$ are reported in table 1 . For the uncoupled case, we consider that the gas is transparent $\left(\mathcal{P}^{\text {rad }}=0\right)$ but that the wall emissivities are the same as in the coupled case (isothermal walls are black, adiabatic vertical walls are perfectly reflecting). There is thus a radiative flux $q_{\text {rad }}$ exchanged between the two black isothermal walls, which does not vary with $z$ because the radiation field does not interact with the flow field (the radiating walls are isothermal).

The conductive flux slightly increases with radiation coupling (the Nusselt number at the wall is 16.66 with radiation and 16.24 without radiation), both at the wall and in the core of the cavity. The convective flux however significantly increases in coupled calculations, especially in the core of the cavity (about $30 \%$ ). In the coupled case, there 

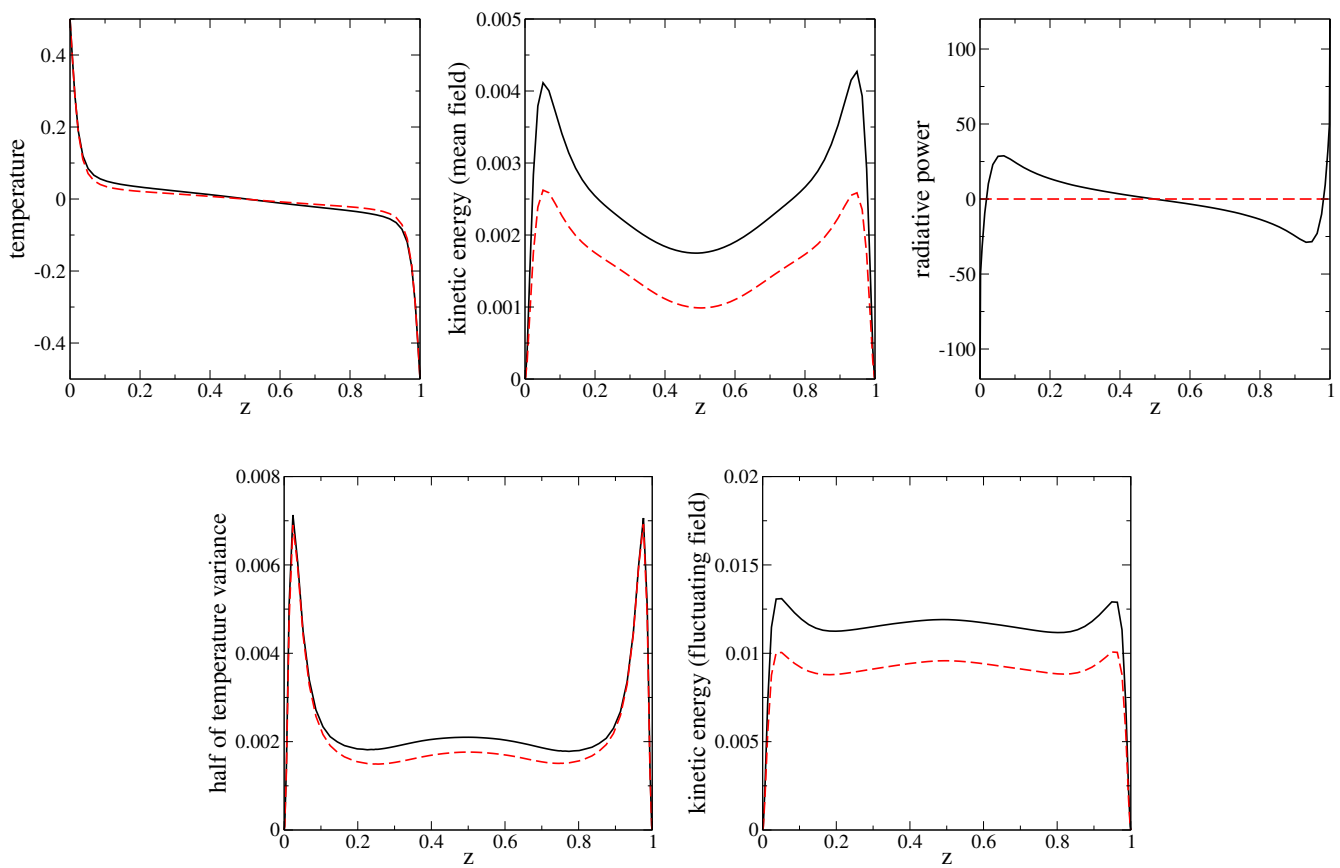

Figure 2. From left to right, top to bottom: mean temperature $\langle\theta\rangle$, mean kinetic energy $0.5\langle\boldsymbol{u}\rangle \cdot\langle\boldsymbol{u}\rangle$, mean radiative power $\left\langle\mathcal{P}^{\text {rad }}\right\rangle$, half temperature variance $0.5\left\langle\theta^{\prime} \theta^{\prime}\right\rangle$ and turbulent kinetic energy $0.5\left\langle\boldsymbol{u}^{\prime} \cdot \boldsymbol{u}^{\prime}\right\rangle$ for coupled (black) and uncoupled (red-dashed) results. Results are averaged over horizontal planes.

\begin{tabular}{lccccccccccc} 
Wall & $q_{\text {cond }}$ & $q_{\text {conv }}$ & $q_{\text {rad }}$ & $q_{\text {tot }}$ & & Core & $q_{\text {cond }}$ & $q_{\text {conv }}$ & $q_{\text {rad }}$ & $q_{\text {tot }}$ \\
\cline { 1 - 6 } Coupled & 16.66 & 0 & 120.82 & 137.48 & & Coupled & 0.12 & 21.62 & 115.77 & 137.51 \\
Uncoupled & 16.24 & 0 & 125.05 & 141.29 & & Uncoupled & 0.08 & 16.14 & 125.05 & 141.27
\end{tabular}

TABLE 1. Energy budget at the wall and in the core of the cavity. Wall values are obtained by averaging top $z=1$ and bottom $z=0$ wall values. Core values correspond to $z=0.5$.

is actually an energy transfer between radiation and convection outside the boundarylayers: in the lower half of the cavity, the gas absorbs radiation, the radiative flux decreases with $z$ and the convective flux increases accordingly while in the upper half of the cavity, the gas emits radiation, the radiative flux increases with $z$ and the convective flux decreases accordingly. This convection enhancement through radiation is not possible in the uncoupled case (the radiative flux is constant) where energy transfer is restricted by boundary-layers near the walls. When considering a participating medium, it can be noticed that the radiative flux at the wall is smaller (120.79 with radiation coupling and 125.05 without) because gas absorption reduces the radiative exchange between the isothermal walls. The total energy flux is thus also weaker in the coupled case. The total energy flux values at the wall and in the core are consistent although there is a small imbalance that is due to time averaging. 
Reduced-order modelling of radiative transfer effects on Rayleigh-Bénard convection 9
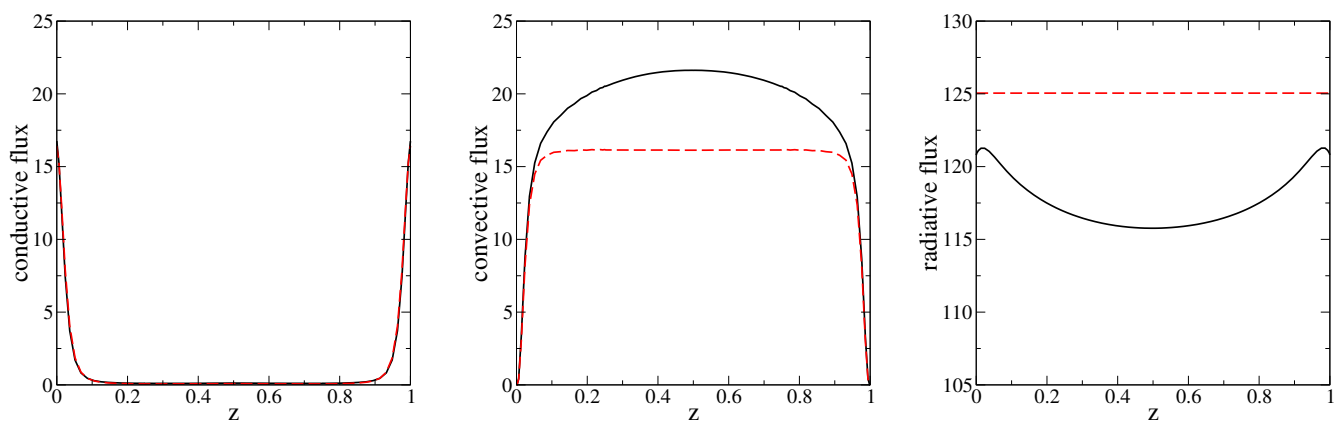

Figure 3. Conductive flux $q_{\text {cond }}$, convective flux $q_{\text {conv }}$ and radiative flux $q_{\text {rad }}$ as defined in equation (3.1) for coupled (black) and uncoupled (red-dashed) cases. Results are averaged over horizontal planes.

\subsection{Flow reorientations}

The unsteady dynamics of the flow in a Rayleigh Bénard cubic cell without radiative transfer is characterized by low-frequency reorientations of the Large Scale Circulation (LSC) (Bai et al. 2016; Foroozani et al. 2017; Vasiliev et al. 2019). The LSC is made of a dominant roll that convects heat from the hot wall to the cold wall and lies in one of the two diagonal planes $x=y$ or $x=1-y$ with a clockwise or anticlockwise motion (namely four quasi-stable states). A reorientation between two quasi-stable states correspond to a sudden rotation of the LSC of $\pi / 2$ around the vertical axis. Reorientations can be monitored with the time evolution of the $x$ and $y$ components of the global angular momentum with respect to the centre of the cavity $\boldsymbol{r}_{0}$

$$
\boldsymbol{L}=\int\left(\boldsymbol{r}-\boldsymbol{r}_{0}\right) \times \boldsymbol{u} d \boldsymbol{r},
$$

which is plotted in figure 4 for both coupled and uncoupled calculations. It can be seen that the coupling with radiation does not change the overall dynamics of reorientations. Both components $L_{x}$ and $L_{y}$ display quasi-stable periods with moderate oscillations around a mean value separated by abrupt aperiodic sign switches corresponding to reorientations. The dynamics seems however more chaotic in the coupled case with several reorientation attempts that are quick passing through a new stable state before returning to the initial stable state. The coupled case is also characterised by a larger disequilibrium in the time spent in each of the four quasi-stable flow states. This disequilibrium clearly appears in the histograms $\left(L_{x}-L_{y}\right)$ provided in figure 4 with a prevalence of the state $\left(L_{x}>0 ; L_{y}<0\right)$ to the detriment of the state $\left(L_{x}<0 ; L_{y}>0\right)$. In the uncoupled case, the imbalance between the four states is much weaker. It is also worth noting that the flow spends more time in the vertical planes $x=0.5$ or $y=0.5$ (corresponding to $L_{x}=0$ or $\left.L_{y}=0\right)$ in the coupled case compared to the uncoupled case.

The reorientation frequency can be estimated by tracking the zeros of a filtered time evolution of $L_{x}$ and $L_{y}$ to avoid small scale noise. We find a reorientation frequency of $1.65 \times 10^{-3}$ for coupled results and of $1.45 \times 10^{-3}$ for uncoupled results. Reorientations seem to be more frequent with radiation although there is some uncertainty in the frequency values given the limited integration time. We can actually infer two competing mechanisms which may affect reorientations in the coupled case: on the one hand, the higher rotation velocity of the LSC tends to stabilize the flow around a stable state, and on the other hand, higher velocity fluctuations tend to promote rotation of the LSC and reorientation events. Finally, a high frequency is noticeable in the time evolution of figure 4 . It corresponds to the rotation frequency of the LSC. It can be estimated by 

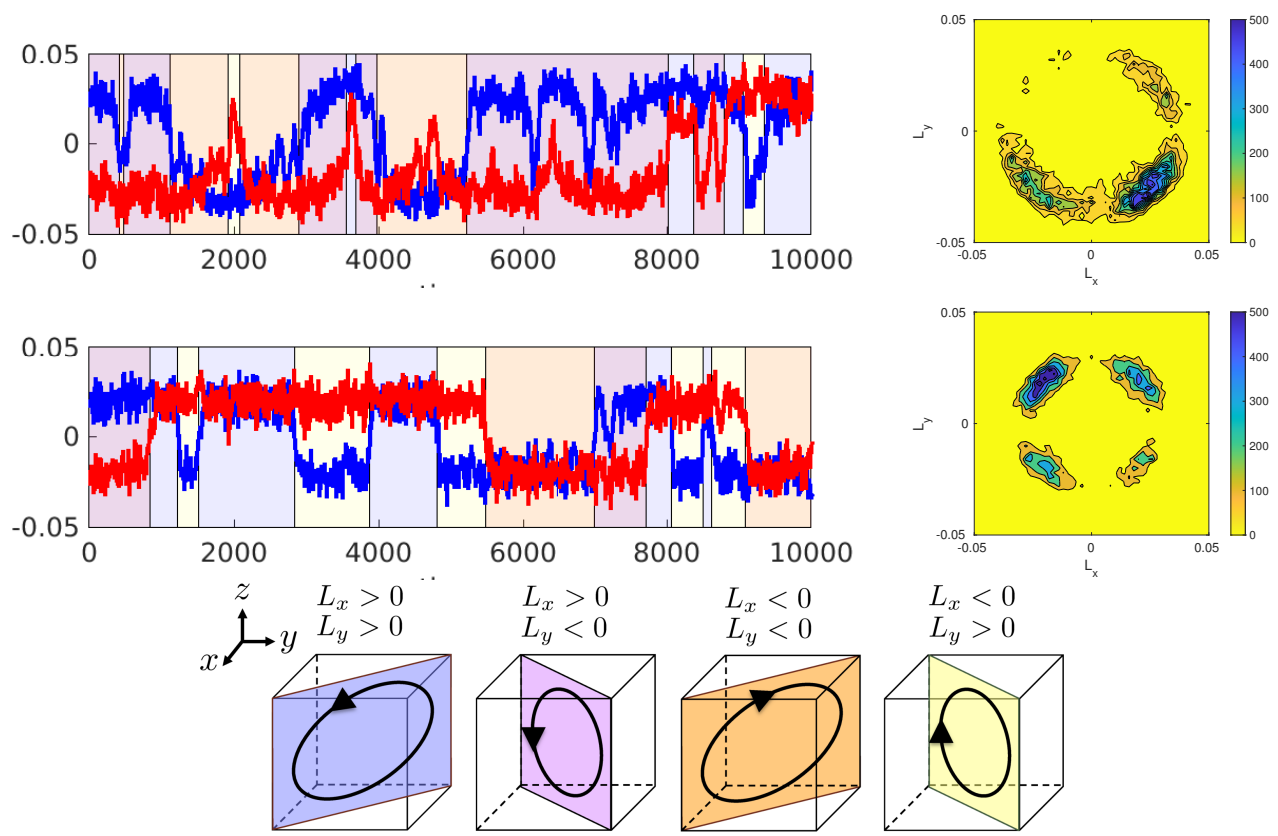

Figure 4. Time evolution of the three components of the angular momentum (left) and histograms of the $x$ and $y$ components (right). Coupled calculations (top) and uncoupled calculations (bottom). Time intervals are coloured according to their associated quasi-stable state.

$f_{c}=1 / T_{c}=0.0233$ in the coupled case using a reference ellipsoid path length 3.85 and a reference velocity $w_{\text {ref }}=0.0898$. The LSC rotates faster than in the uncoupled case $\left(f_{c}=0.0205, w_{\text {ref }}=0.0788\right)$, this increase of about $13 \%$ being consistent with the global kinetic energy increase of $30 \%$.

\subsection{POD analysis}

A POD analysis of coupled flow results has been carried out. It consists in searching for a basis of spatial modes or empirical eigenfunctions $\phi_{n}(\boldsymbol{r})$ that optimally represent the flow field $\boldsymbol{U}(\boldsymbol{r}, t)=\{\boldsymbol{u}, \gamma \theta\}$, where $\gamma$ is a scaling factor to be specified. These spatial modes are orthonormal and their amplitude $a_{n}(t)$ vary in time. One has

$$
\boldsymbol{U}(\boldsymbol{r}, t)=\sum_{n=1}^{\infty} a_{n}(t) \phi_{n}(\boldsymbol{r}) .
$$

The POD modes are hierarchically organised according to their energy content $\lambda_{n}$ and their amplitudes are statistically uncorrelated, namely $\left\langle a_{n}(t) a_{m}(t)\right\rangle=\lambda_{n} \delta_{n m}$ where $\delta_{n m}$ is the Kronecker symbol. The objective is to restrict the decomposition (3.3) to a few modes with the largest eigenvalues so that the flow dynamics can be analyzed in a loworder subspace able to capture the most of the energy of the field $\boldsymbol{U}(\boldsymbol{r}, t)$. The POD spatial modes $\phi_{n}(\boldsymbol{r})$ are solution of the following eigenvalue problem (Berkooz et al. 1993)

$$
\int \sum_{k=1}^{4}\left\langle U^{m}(\boldsymbol{r}, t) U^{k}\left(\boldsymbol{r}^{\prime}, t\right)\right\rangle \phi_{n}^{k}\left(\boldsymbol{r}^{\prime}\right) d \boldsymbol{r}^{\prime}=\lambda_{n} \phi_{n}^{m}(\boldsymbol{r}),
$$



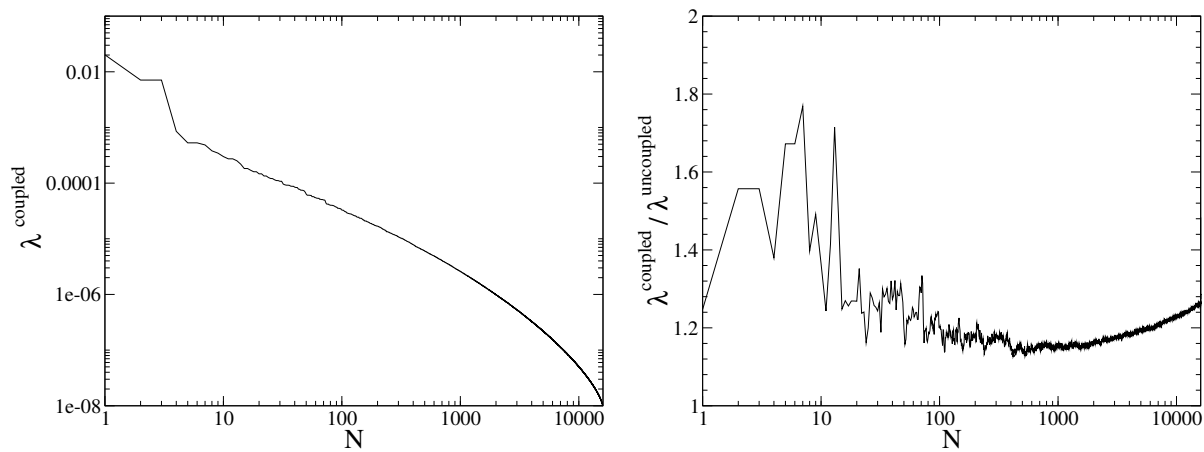

FiguRE 5. POD eigenspectrum obtained from coupled simulations (left) and POD eigenspectrum ratio between coupled and uncoupled results (right).

which is solved in practice using the method of snapshots (Sirovich 1987). A statistical set of 1,000 snapshots $\boldsymbol{U}\left(\boldsymbol{r}, t_{i}\right)$ is extracted from the coupled DNS at discrete times $t_{i}$ with a constant sampling period of 10 dimensionless time units (thus covering the whole DNS time sequence of 10,000 time units). In order to improve the convergence of the POD method, we have built an enlarged snapshot set, obtained by the action of the symmetry group of the problem on the original snapshot set (Holmes et al. 1996). The symmetry group (including the $S_{z}$ symmetry see section 2.1) contains 16 elements and this allows us to multiply the number of snapshots by a factor of 16 to obtain a final snapshot set of 16,000 samples. Finally, the rescaling factor $\gamma$ used to combine temperature and velocity field is chosen so that each field has the same energy (Podvin \& Le Quéré 2001)

$$
\gamma^{2}=\left\langle\frac{\int \boldsymbol{u}(\boldsymbol{r}, t) \cdot \boldsymbol{u}(\boldsymbol{r}, t) d \boldsymbol{r}}{\int \theta^{2}(\boldsymbol{r}, t) d \boldsymbol{r}}\right\rangle .
$$

Because velocity fluctuations are proportionately larger than temperature fluctuations in coupled calculations, this factor is greater when coupling with radiation: we obtain $\gamma^{\text {coupled }}=1.421$ and $\gamma^{\text {uncoupled }}=1.303$. In the following, the POD analysis of coupled DNS will be compared to the POD analysis of uncoupled DNS discussed by Soucasse et al. (2019).

The POD eigenvalue spectrum is shown in figure 5. The eigenvalue decay is rather slow owing to the 3-D and turbulent nature of the flow, even so the first three modes contain $62 \%$ of the total energy. Is also given in figure 5 the ratio between the eigenvalue spectrum obtained from coupled simulations and the eigenvalue spectrum obtained from uncoupled simulations. All eigenvalues are higher in the coupled case, with larger eigenvalue ratios for the low-order modes $n \leqslant 20$, a minimum value of 1.1 around $n=1000$ followed by a slow increase for the higher-order modes. The shape of the spectrum ratio can be interpreted if we consider that mode ordering roughly corresponds to a ranking of the eigenfunctions in terms of a characteristic spatial scale, the low-order modes being associated with the largest spatial scales and the high-order modes being associated with the smallest spatial scales. We have seen that radiation-convection energy transfer outside the boundary-layers reinforces the large-scale flow structures. This supplementary energy compared to the uncoupled case is transported towards smaller scales of the turbulent spectrum but is also dissipated because of radiative damping of thermal fluctuations. Radiative damping rather affects the intermediate spatial scales as molecular diffusion prevails for the smallest spatial scales, which may explain the decrease of the ratio for the intermediate modes. 

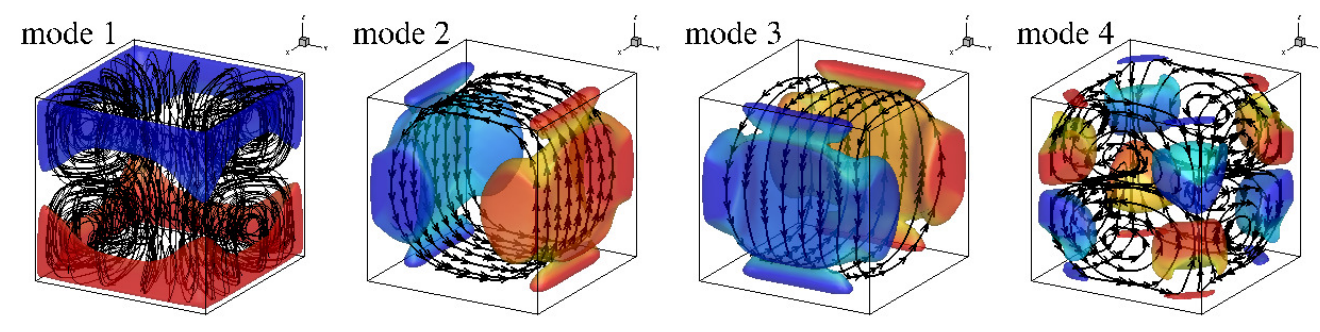

$\mathrm{a}_{3}$
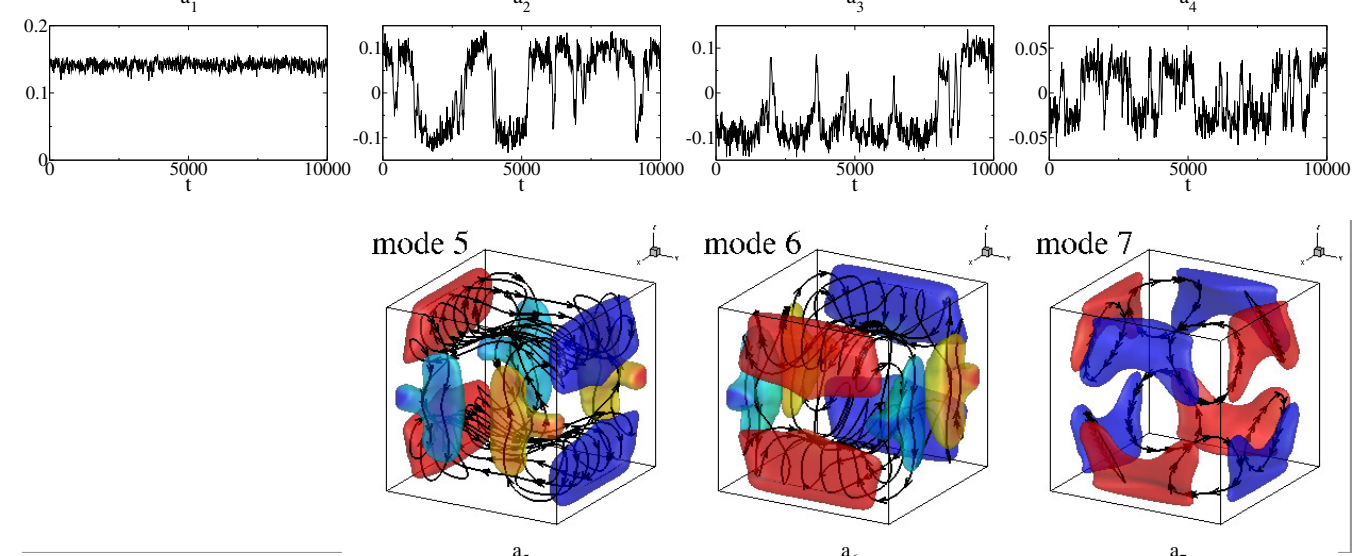

$\mathrm{a}_{5}$

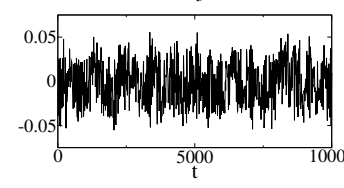

$\mathrm{a}_{6}$
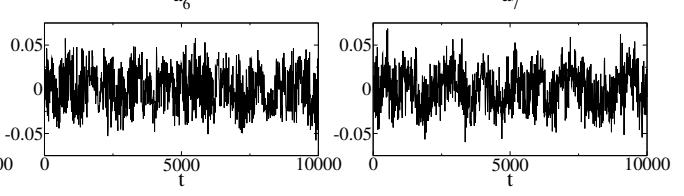

Figure 6. First seven POD modes $\phi_{n}(\boldsymbol{r})$ obtained from coupled simulations with associated amplitude $a_{n}(t)$. Streamlines and iso-surfaces of convective heat flux $\phi_{n}^{\theta} \phi_{n}^{w}=0.25$ coloured by mode temperature. Colourmap for mode temperature ranges from -0.5 (blue) to 0.5 (red).

The first seven POD modes are shown in figure 6, together with their associated amplitude $a_{n}(t)$ in the coupled DNS time sequence. Isovalues of the convective heat flux $\phi_{n}^{\theta} \phi_{n}^{w}$ coloured by mode temperature, as well as streamlines, are displayed to highlight the mechanical and thermal structures. A remarkable feature is that these first seven eigenfunctions are nearly identical to the first seven eigenfunctions obtained without radiation coupling, although the associated eigenvalues are different. Namely, the projection matrix $\boldsymbol{P}$ from the POD coupled basis $\mathcal{B}^{\text {coupled }}=\left\{\phi_{1}, \phi_{2}, \cdots, \phi_{7}\right\}^{\text {coupled }}$ onto the POD uncoupled basis $\mathcal{B}^{\text {uncoupled }}=\left\{\phi_{1}, \phi_{2}, \cdots, \phi_{7}\right\}^{\text {uncoupled }}$ is very close to the identity matrix: we get $\|\boldsymbol{P}-\boldsymbol{I}\|_{F}=0.052$, if $\|\cdot\|_{F}$ denotes the Frobenius norm. This result will be key for the derivation of an a priori reduced-order model of radiative transfer effects (see section 4.2.2). We briefly recall below the physical meaning of these modes.

The first mode corresponds to the mean flow: its amplitude is nearly constant and oscillates around a mean value $a_{1}^{e q}=\sqrt{\lambda_{1}}$. The velocity field is made of two counterrotating torus-like structures and the temperature field is vertically stratified from the bottom hot wall to the top cold wall. Modes 2 and 3 form a pair of degenerated modes: $\lambda_{2}=\lambda_{3}$ and $\phi_{2}(\boldsymbol{r})$ and $\phi_{3}(\boldsymbol{r})$ are identical after a rotation of $\pi / 2$ around the $z$ axis. They are made of a large-scale single roll around the $x$ axis (mode 2) or the $y$ axis (mode 3 ) and are referred to as LSC modes. Their time evolution is correlated with the $x$ and $y$ 

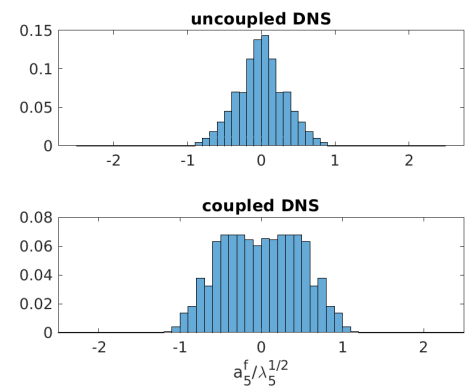

Figure 7. Histogram of the filtered amplitude $a_{5}^{f}$ in the uncoupled and coupled DNS; the amplitudes are filtered with a moving average of 100 dimensionless time units

components of the angular momentum (see figure 4) and displays aperiodic sign switches (reorientations) between two quasi-stable states where the amplitude oscillates around a mean value $a_{2 / 3}^{e q}= \pm \sqrt{\lambda_{2 / 3}}$. Modes 2 and 3 have therefore to be combined to form the quasi-stable diagonal states observed in the simulation. Mode 4 is an 8 -roll mode that transports fluid from one corner to the other and strengthens the circulation along the diagonal. Its time evolution is correlated with the product $a_{2}(t) a_{3}(t)$ and displays abrupt sign switches around equilibrium values $a_{4}^{e q}=\operatorname{sgn}\left(a_{2}^{e q} a_{3}^{e q}\right) \sqrt{\lambda_{4}}$. Its sign actually indicates the diagonal plane of the LSC: $a_{4}>0$ means the LSC lies in the plane $x=1-y$; $a_{4}<0$ means the LSC lies in the plane $x=y$. Modes 5 and 6 form another pair of degenerated modes and are referred to as boundary-layer modes. They are made of two longitudinal co-rotating structures around the $x$ axis (mode 5) or the $y$ axis (mode 6) and connect the core of the cell with the horizontal boundary-layers. Modes 2 and 5 (respectively modes 3 and 6 ) possess the same symmetries and strongly interact together. Although the time evolution of amplitudes $a_{5}(t)$ and $a_{6}(t)$ seems noisy and chaotic, a moving average over 90 dimensionless time units shows a non-zero equilibrium contribution during quasi-stable states, with sign switches during reorientations such that $\operatorname{sgn}\left(a_{5 / 6}^{e q}\right)=-\operatorname{sgn}\left(a_{2 / 3}^{e q}\right)$. As figure 7 shows, this contribution is much lower than the standard deviation $a_{5 / 6}^{e q}= \pm \eta \sqrt{\lambda_{5 / 6}}$, in the uncoupled case (an estimated value of $\eta$ was about 0.2 ), but it is higher in the coupled case, with a value of $\eta=0.35$. This means that the connection between the roll and the boundary layer modes is much stronger in the coupled case. Finally, mode 7 is a corner-roll mode which favours planar LSC (in planes $x=0.5$ or $y=0.5$ ) rather than diagonal LSC (in planes $x=y$ and $x=1-y$ ). It has a destabilizing effect on the quasi-stable states, although its temporal evolution does not show any specific pattern during reorientations.

A last comment can be made regarding the thermal or mechanical nature of the POD eigenfunctions. Though temperature and velocity fields have been combined to perform the POD analysis, the mechanical and thermal weight associated with each POD mode $\phi_{n}=\left\{\boldsymbol{\phi}_{n}^{u}, \gamma \phi_{n}^{\theta}\right\}$ can be retrieved according to

$$
\left\|\phi_{n}\right\|^{2}=\left\|\phi_{n}^{u}\right\|^{2}+\gamma^{2}\left\|\phi_{n}^{\theta}\right\|^{2}=1 .
$$

Table 2 shows that the relative energy weights $\left\|\phi_{n}^{u}\right\|^{2}$ and $\gamma^{2}\left\|\phi_{n}^{\theta}\right\|^{2}$ are not the same for each mode. Mode 1, which possesses the mean thermal stratification, is a thermal mode while modes 2 to 7 are rather mechanical modes. One can also notice that the POD mode ranking would differ if one uses a mechanical energy criterion $\left(\lambda_{n}\left\|\phi_{n}^{u}\right\|^{2}\right)$ or a thermal energy criterion $\left(\lambda_{n} \gamma^{2}\left\|\phi_{n}^{\theta}\right\|^{2}\right)$. This has been further confirmed by performing a separate POD analysis of velocity and temperature fields: the same dominant structures 


$\begin{array}{lccccc}\text { Mode } n & 1 & 2,3 & 4 & 5,6 & 7 \\ \lambda_{n} & 2.0 \times 10^{-2} & 7.1 \times 10^{-3} & 8.5 \times 10^{-4} & 5.3 \times 10^{-4} & 4.8 \times 10^{-4} \\ \left\|\phi_{n}^{u}\right\|^{2} & 0.13 & 0.76 & 0.82 & 0.69 & 0.64 \\ \gamma^{2}\left\|\phi_{n}^{\theta}\right\|^{2} & 0.87 & 0.24 & 0.18 & 0.31 & 0.36\end{array}$

TABLE 2. Thermal and mechanical content of the first seven POD eigenfunctions given by partial norms $\left\|\phi^{u}\right\|^{2}$ and $\gamma^{2}\left\|\phi^{\theta}\right\|^{2}$.

are obtained but they are not ranked exactly in the same way compared to the combined case.

To sum up, POD analysis shows that the combined mechanical and thermal fluctuating energy increases, and that the ratio of mechanical to thermal energy increases in the presence of radiation, although the POD most energetic structures are not significantly modified.

\section{Reduced-order modelling}

\subsection{POD-based model with radiative terms}

A POD-based reduced-order mode including radiation effects can be derived using Galerkin projection of momentum and energy balance with radiation (2.2)-(2.3) onto a POD basis set. Using decomposition (3.3) this yields a system of ordinary differential equations of the form

$$
\frac{d a_{n}(t)}{d t}=\left(L_{n m}^{B}+L_{n m}^{D}+L_{n m}^{R}\right) a_{m}(t)+Q_{n m p} a_{m}(t) a_{p}(t)+T_{n}(t)
$$

where $L_{n m}^{B}, L_{n m}^{D}$ and $L_{n m}^{R}$ are linear contributions associated with buoyancy, diffusion and radiation respectively, $Q_{n m p}$ are quadratic contributions associated with advection of momentum and energy (definitions of these quantities are provided in appendix B). $T_{n}(t)$ is a closure term for taking into account the effect of the truncation.

\subsubsection{Radiative terms}

Radiative transfer effects can be considered as a linear contribution owing to the weak temperature differences. The radiative emission in $k_{j} w_{j} \sigma T^{4} / \pi$ in the radiative transfer equation (2.7) can be linearised around the mean temperature $T_{0}$. Because the radiative transfer equation is linear, we can then use the superposition principle and write the radiative intensity as a sum of partial intensities associated with each term of the POD decomposition of the temperature field. We are therefore able to define a modal-radiative power $\mathcal{P}_{n}^{\mathrm{rad}}(\boldsymbol{r})$, corresponding to the radiative response to a temperature eigenfunction $\phi_{n}^{\theta}(\boldsymbol{r})$ and such that

$$
\mathcal{P}^{\mathrm{rad}}(\boldsymbol{r}, t)=\sum_{n} a_{n}(t) \mathcal{P}_{n}^{\mathrm{rad}}(\boldsymbol{r}) .
$$

Details of the derivation of this modal-radiative power are given in appendix A. Figure 8 shows the modal-radiative power $\mathcal{P}_{n}^{\text {rad }}$ associated with the first seven modes. It is worth noting that $\mathcal{P}_{n}^{\text {rad }}$ possesses the same symmetries as the associated temperature eigenfunction $\phi_{n}^{\theta}$. Once $\mathcal{P}_{n}^{\text {rad }}$ is determined, the radiation matrix $L^{R}$ can be computed according to

$$
L_{n m}^{R}=\int \frac{\gamma^{2}}{\sqrt{R a}} \mathcal{P}_{m}^{\mathrm{rad}}(\boldsymbol{r}) \phi_{n}^{\theta}(\boldsymbol{r}) d \boldsymbol{r}
$$


Reduced-order modelling of radiative transfer effects on Rayleigh-Bénard convection 15
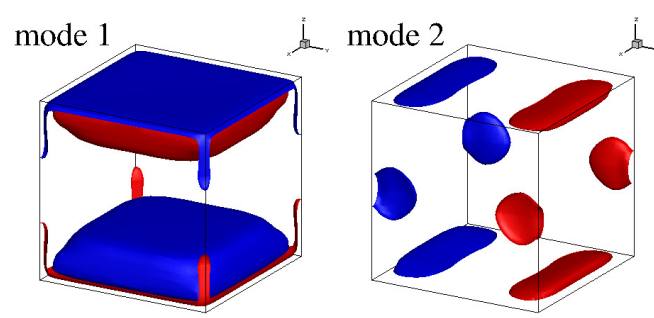

选, mode 3

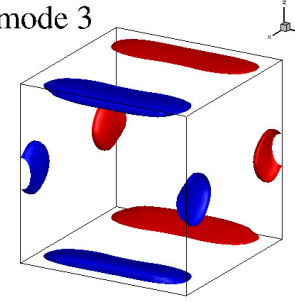

I. mode 4
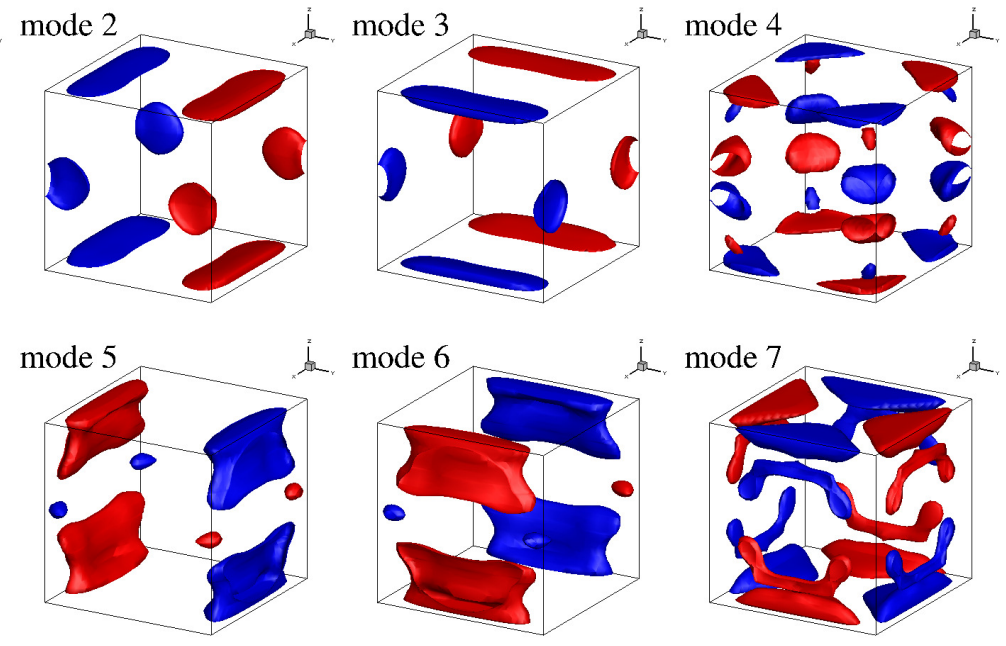

FIGURE 8. Isovalues of modal-radiative power $\mathcal{P}_{m}^{\text {rad }}(\boldsymbol{r})= \pm 2.5$ for the first seven modes.

Values of the coefficients are reported in appendix B for the coupled temperature eigenfunctions $\phi_{n}^{\theta}(\boldsymbol{r})$ presented in section 3.3. The radiation matrix is essentially diagonal with two off-diagonal terms corresponding to a linear coupling between modes 2 and 5 , and between modes 3 and 6 (these pairs of modes possess the same symmetries). The coefficients $L_{n m}^{R}$ have the same sign as the coefficients $L_{n m}^{D}$ of the diffusion matrix, which means that radiation will affect the reduced-order model as molecular diffusion does, and are such that $L_{n m}^{R} \sim 0.1 L_{n m}^{D}$ (except for $\left.L_{11}^{R}\right)$.

\subsubsection{Closure}

Following Podvin \& Sergent (2015), the closure term is modelled as an equivalent dissipation term, expressed as a combination of linear and cubic terms, and by the addition of noise such that

$$
T_{n}(t)=L_{n m}^{A}\left(1-\frac{1}{\langle k\rangle} \sum_{p \geqslant 2}\left|a_{p}(t)\right|^{2}\right) a_{m}(t)+\epsilon(t)
$$

where $L_{n m}^{A}$ is an adjustable parameter, $\langle k\rangle$ is the temporal average of the energy of the fluctuating modes in the truncation and $\epsilon(t)$ is a random noise perturbation of amplitude $\sigma$. If the coefficients $L_{n m}^{A}$ are not known before-hand, it is possible to determine them from the observed dynamics, as was done in Soucasse et al. (2019). We will get back to this point in section 4.2.1. Assuming that the closure law derived without radiation remains valid in the presence of radiation, the final form of the model is thus

$$
\frac{d a_{n}(t)}{d t}=\left(L_{n m}^{T}-\frac{L_{n m}^{A}}{\langle k\rangle} \sum_{p \geqslant 2}\left|a_{p}(t)\right|^{2}\right) a_{m}(t)+Q_{n m p} a_{m}(t) a_{p}(t)+\epsilon(t)
$$

where $L^{T}=L^{B}+L^{D}+L^{A}+L^{R}$ denotes the total linear contribution.

In Soucasse et al. (2019) we derived a POD-based dynamical system to describe the evolution of the amplitudes of the largest POD modes in the same configuration in the absence of radiation. This previous model will be referred to as no-radiation model in the remainder of the paper. In the following, we develop two different models including radiation effects. 


\begin{tabular}{|c|c|c|c|c|c|c|c|c|c|c|c|c|c|}
\hline \multicolumn{7}{|c|}{ no-radiation model } & \multicolumn{7}{|c|}{ observed model } \\
\hline $\begin{array}{l}L_{n m}^{M} \\
n=2\end{array}$ & $\begin{array}{l}m=2 \\
-0.044\end{array}$ & $m=3$ & $m=4$ & $\begin{array}{c}m=5 \\
0.043\end{array}$ & $m=6$ & $m=7$ & $\begin{array}{l}L_{n m}^{M} \\
n=2\end{array}$ & $m=2$ & $m=3$ & $m=4$ & $m=5$ & $m=6$ & $m=7$ \\
\hline$n=3$ & & -0.044 & & & 0.043 & & $n=3$ & & -0.043 & & & 0.024 & \\
\hline$n=4$ & & & -0.136 & & & & $n=4$ & & & -0.187 & & & \\
\hline$n=5$ & -0.028 & & & -0.143 & & & $n=5$ & -0.030 & & & -0.120 & & \\
\hline$n=6$ & & -0.028 & & & -0.143 & & $n=6$ & & -0.030 & & & -0.120 & \\
\hline$n=7$ & & & & & & -0.068 & $n=7$ & & & & & & -0.047 \\
\hline
\end{tabular}

TABLE 3 . Time-average of the unresolved terms $L^{M}=L^{A}\left(1-\sum_{n=2}^{7} \lambda_{n} /\langle k\rangle\right)$ in the model left) without radiation right) with radiation.

- The first model, called the observed model, is developed from direct observation of the DNS with radiation. Parameters $L^{B}, L^{D}, L^{R}$ and $Q$ are computed from coupled POD eigenfunctions and parameter $L^{A}$ is adjusted based on the known amplitude of the modes at equilibrium (see section 4.2.1).

- The second model, called the predicted model, represents an a priori attempt to predict radiative transfer effects from uncoupled DNS data. Parameters $L^{B}, L^{D}, Q$ and $L^{A}$ are taken from the no-radiation model (Soucasse et al. 2019) and parameter $L^{R}$ is computed from uncoupled POD eigenfunctions (section 4.2.2).

We actually restrict the expansion to the first seven modes and take the first mode (mean flow) as constant so that the dimension of the reduced-order model is equal to six.

\subsection{Observed and predicted models}

\subsubsection{Observed model}

To determine the adjustable parameters $L_{n m}^{A}$ of the observed model, we use information about the POD amplitudes extracted from the DNS with radiation. Namely, we require that the diagonal states be fixed points of the dynamical system $a_{n}^{e q}$, which leads to a balance between the global linear contribution and the quadratic terms. Equilibrium values are taken such that $a_{1}^{e q}=\sqrt{\lambda_{1}}, a_{2 / 3}^{e q}= \pm \sqrt{\lambda_{2 / 3}}, a_{4}^{e q}=\operatorname{sgn}\left(a_{2}^{e q} a_{3}^{e q}\right) \sqrt{\lambda_{4}}, a_{5 / 6}^{e q}=$ $-\operatorname{sgn}\left(a_{2 / 3}^{e q}\right) \eta \sqrt{\lambda_{5 / 6}}, a_{7}^{e q}=0$, where $\eta=0.35$. The values of the time averaged closure terms are given in table 3 . The values are close to those obtained in the case without radiation, except for an increase in mode 4. This suggests that the nature of energy transfer from large scales to small scales remains unaffected. The other parameters of the observed model $\left(L_{n m}^{B}, L_{n m}^{D}, L_{n m}^{R}\right.$ and $\left.Q_{n m p}\right)$ are directly computed from coupled POD eigenfunctions and values are reported in appendix $B$.

In order to estimate the effect of the truncation of the radiation term $\sum_{m} L_{n m}^{R} a_{m}(t)$ in the model (4.5), we have computed the following radiation truncation error

$$
e_{n}=\frac{\left\|\frac{\gamma^{2}}{\sqrt{R a}} \int \mathcal{P}^{\mathrm{rad}}\left(\boldsymbol{r}, t_{i}\right) \phi_{n}^{\theta}(\boldsymbol{r}) d \boldsymbol{r}-\sum_{m=1}^{7} L_{n m}^{R} a_{m}\left(t_{i}\right)\right\|}{\left\|\frac{\gamma^{2}}{\sqrt{R a}} \int \mathcal{P}^{\mathrm{rad}}\left(\boldsymbol{r}, t_{i}\right) \phi_{n}^{\theta}(\boldsymbol{r}) d \boldsymbol{r}\right\|}
$$

where $\mathcal{P}^{\mathrm{rad}}\left(\boldsymbol{r}, t_{i}\right)$ is the radiative power of snapshot $i$ at discrete time $t_{i}, a_{m}\left(t_{i}\right)$ is the associated POD coefficient extracted from the DNS, and the norm is defined such that $\left\|f\left(t_{i}\right)\right\|=\sqrt{\sum_{i} f\left(t_{i}\right)^{2}}$. This quantity is equal to $0.13,0.28,0.20$ and 0.31 for modes $2 / 3$, $4,5 / 6$ and 7 respectively, showing that the radiation contribution of unresolved scales is small but not negligible. This is not surprising given that the first seven modes only contain $60 \%$ of the total energy. We can however infer that the form of the closure law still holds, given that radiation acts as a dissipation term in the model. We will thus assume 
that the effect of the truncation of the radiation term is captured by the adjustable parameter $L^{A}$.

\subsubsection{Predicted model}

The second model attempts to predict the change in the energy level of the modes directly from the uncoupled simulation results and the computation of the radiation terms. We assume that the eigenfunctions are the same as in the uncoupled case, that the modelled terms do not change and that the value of $\gamma$ is not modified. The only modification of the model is therefore due to the radiative terms which are computed from the uncoupled POD eigenfunctions.

Let $r_{i}^{0}$ be the equilibria in the uncoupled case. Owing to the symmetry between $x$ and $y$, it is sufficient to consider the fixed point $r_{2}^{0}=r_{3}^{0}=\sqrt{\lambda_{2 / 3}^{0}}, r_{5}^{0}=r_{6}^{0}=-\eta \sqrt{\lambda_{5 / 6}^{0}}$ $(\eta=0.2), r_{4}^{0}=\sqrt{\lambda_{4}^{0}}$, where $\lambda_{n}^{0}$ denotes the uncoupled POD eigenvalue of mode $n$. In the uncoupled case at equilibrium, we can derive the following relations for modes $2 / 3,5 / 6$ and 4

$$
\begin{gathered}
L_{22}^{B}+L_{22}^{D}+L_{22}^{M}+Q_{212} r_{1}^{0}+Q_{234} r_{4}^{0}=0, \\
L_{52}^{B}+L_{52}^{D}+L_{52}^{M}+Q_{512} r_{1}^{0}+Q_{534} r_{4}^{0}=0, \\
\left(L_{44}^{B}+L_{44}^{D}+L_{44}^{M}+Q_{414} r_{1}^{0}\right) r_{4}^{0}+Q_{423}\left(r_{2}^{0}\right)^{2}+2 Q_{425} r_{2}^{0} r_{5}^{0}+Q_{456}\left(r_{5}^{0}\right)^{2}=0,
\end{gathered}
$$

where $L^{M}=L^{A}\left(1-\sum_{n=2}^{7} \lambda_{n} /\langle k\rangle\right)$ is the equilibrium (time-averaged) closure term in the no-radiation model (see table 3 ) and parameters $L^{B}, L^{D}$ and $Q$ are computed from uncoupled POD eigenfunctions. Note that in equations (4.7) and (4.8) associated with modes 2 and 5 , we assume that diagonal and off-diagonal linear contributions are balanced independently.

We now determine how inclusion of the radiation term leads to changes in the energy of the modes at equilibrium. The unknown equilibria in the radiatively coupled case are denoted $r_{1}, r_{2}, r_{3}=r_{2}, r_{4}, r_{5}, r_{6}=r_{5}, r_{7}=0$. The predicted equilibrium relations with radiation write

$$
\begin{gathered}
L_{22}^{B}+L_{22}^{D}+L_{22}^{R}+L_{22}^{M}+Q_{212} r_{1}+Q_{234} r_{4}=0, \\
L_{52}^{B}+L_{52}^{D}+L_{52}^{R}+L_{52}^{M}+Q_{512} r_{1}+Q_{534} r_{4}=0, \\
\left(L_{44}^{B}+L_{44}^{D}+L_{44}^{R}+L_{44}^{M}+Q_{414} r_{1}\right) r_{4}+Q_{423} r_{2}^{2}+2 Q_{425} r_{2} r_{5}+Q_{456} r_{5}^{2}=0 .
\end{gathered}
$$

Subtracting the two systems, equation by equation, one obtains

$$
\begin{gathered}
Q_{212} r_{1}+Q_{234} r_{4}=-L_{22}^{R}+Q_{212} r_{1}^{0}+Q_{234} r_{4}^{0}, \\
Q_{512} r_{1}+Q_{534} r_{4}=-L_{52}^{R}+Q_{512} r_{1}^{0}+Q_{534} r_{4}^{0}, \\
Q_{414} r_{1} r_{4}+Q_{423} r_{2}^{2}+2 Q_{425} r_{2} r_{5}+Q_{456} r_{5}^{2} \\
=-L_{44}^{R} r_{4}+Q_{414} r_{1}^{0} r_{4}^{0}+Q_{423}\left(r_{2}^{0}\right)^{2}+2 Q_{425} r_{2}^{0} r_{5}^{0}+Q_{456}\left(r_{5}^{0}\right)^{2} .
\end{gathered}
$$

Equations (4.13) and (4.14) can be solved directly for $r_{1}$ and $r_{4}$. We obtain new values of 0.1578 and 0.028 , representing increases of $22 \%$ and $14 \%$. Equation (4.15) involves $r_{2}$ and $r_{5}$, which are both unknown. We note that it displays a quadratic dependence of $r_{2}$ with respect to $r_{4}$. Moreover, in the uncoupled DNS (also in the coupled DNS), the amplitude of mode 4 is very well correlated with the product $a_{2} a_{3}$ (with a correlation coefficient larger than 0.9), so that $a_{4} \sim C a_{2} a_{3}$. We use this empirical relationship to 


\begin{tabular}{|c|c|c|c|c|c|c|c|}
\hline Mode $n$ & 1 & 2,3 & 4 & & 5,6 & & 7 \\
\hline$\sqrt{\lambda_{n}^{0}}$ (uncoupled DNS) & 0.127 & 0.067 & 0.024 & & 0.017 & & 0.0165 \\
\hline$\sqrt{\lambda_{n}}($ coupled DNS) & $0.141(12 \%)$ & $0.085(25 \%)$ & 0.029 & $(17 \%)$ & 0.023 & $(29 \%)$ & 0.022 \\
\hline$\sqrt{\lambda_{n}^{p}}$ (predicted) & $0.154(22 \%)$ & $0.071 \quad(7 \%)$ & 0.028 & $(14 \%)$ & 0.020 & $(15 \%)$ & $\mathrm{N} / \mathrm{A}$ \\
\hline
\end{tabular}

TABle 4. Amplitudes of the POD eigenvalues $\lambda_{n}^{0}$ and $\lambda_{n}$ in the uncoupled and coupled simulations and predicted energies $\lambda_{n}^{p}$. The value of $\lambda_{5}^{p}$ is extrapolated from $r_{5}$ using $\lambda_{5}^{p}=\lambda_{5}^{0}\left(r_{5} / r_{5}^{0}\right)^{2}$. The relative increase with respect to the uncoupled case is indicated in parentheses.

estimate the increase in $r_{2}$ such that

$$
r_{2}=r_{2}^{0} \sqrt{\frac{r_{4}}{r_{4}^{0}}},
$$

then solve equation (4.15) for $r_{5}$. We obtain an increase of $7 \%$ for $r_{2}$ and of $15 \%$ for $r_{5}$. The values of the predicted energies $\lambda_{n}^{p}$ are reported in table 4 . An estimate of the energy of POD mode $5, \lambda_{5}^{p}$, was made from the new equilibrium value $r_{5}$ by assuming that $\eta$ remains constant, so that $\lambda_{5}^{p}=\lambda_{5}^{0}\left(r_{5} / r_{5}^{0}\right)^{2}$. Another estimate will be provided by integration of the predicted model (see section 4.3 ). The model therefore predicts an increase in the energy of all the modes which is of the order of that observed in the simulation, with an overprediction of the mean mode and an underprediction of the roll modes.

\subsection{Time integration}

All models were integrated for a given noise level $\sigma$ over 100,000 dimensionless time units. The noise signal was the same for all models, but the noise intensities were different. In the no-radiation model, the noise level was $\sigma_{0}=1.2 \times 10^{-3}$. In the observed model, the noise level is determined from the total energy level in the unresolved modes i.e.

$$
\frac{\sigma}{\sigma_{0}}=\frac{\sum_{n>7} \lambda_{n}}{\sum_{n>7} \lambda_{n}^{0}} \sim 1.22 .
$$

The observed model was therefore integrated for relative noise levels $\sigma / \sigma_{0}$ of 1.2 and 1.3. In the predicted model, the noise level is estimated from the new energy level of the resolved modes. Since the transfer to the unresolved scales is assumed to be the same (it is of the form $L^{M} a$, where $L^{M}$ does not change), the noise level was therefore determined using

$$
\frac{\sigma}{\sigma_{0}}=\sqrt{\frac{\sum_{n \leqslant 6} \lambda_{n}^{p}}{\sum_{n \leqslant 6} \lambda_{n}^{0}}} \sim 1.17 .
$$

Results for the predicted model are shown for values of $\sigma / \sigma_{0}$ of 1.1 and 1.2.

Histories for POD amplitudes $a_{2}, a_{3}$ and $a_{4}$ in the observed and predicted models are displayed in figure 9 for the first 10,000 dimensionless time units. It can be seen that the overall dynamics of the coupled simulation (see figure 6) is fairly reproduced: the system spends long time near equilibrium values and experiences fast reorientations ( $\operatorname{sign}$ switch of either $a_{2}$ or $a_{3}$ ); the time evolution of $a_{4}$ is well correlated with the product $a_{2} a_{3}$. The energy of the amplitudes integrated with the different models are reported in table 5. Results for the predicted and the observed models are in good agreement with the simulation (table 4), except for mode 7, the last mode in the truncation, which is overestimated. However, the relative increase in mode 7 appears to be relatively well 

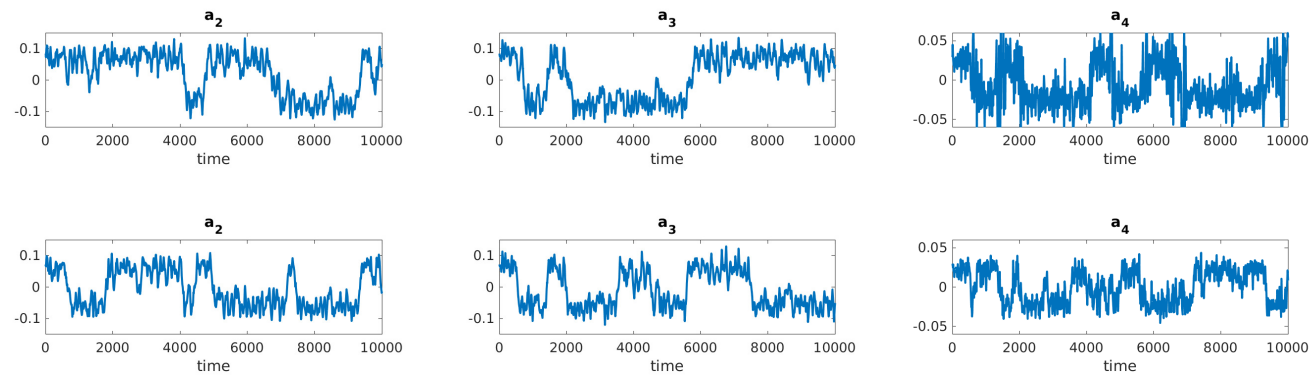

Figure 9. Time evolution of the amplitudes $a_{2}, a_{3}, a_{4}$ from left to right; top row: observed model $\left(\sigma=1.2 \sigma_{0}\right)$; bottom row: predicted model $\left(\sigma=1.2 \sigma_{0}\right)$.

\begin{tabular}{|c|c|c|c|c|c|}
\hline Mode $n$ & 1 & 2,3 & 4 & 5,6 & 7 \\
\hline$\sqrt{\left\langle a_{n}^{2}\right\rangle}\left(\right.$ observed, $\left.\sigma=1.2 \sigma_{0}\right)$ & 0.141 & 0.074 & 0.029 & 0.023 & 0.052 \\
\hline$\sqrt{\left\langle a_{n}^{2}\right\rangle}\left(\right.$ observed, $\left.\sigma=1.3 \sigma_{0}\right)$ & 0.141 & 0.073 & 0.030 & 0.023 & 0.053 \\
\hline$\sqrt{\left\langle a_{n}^{2}\right\rangle}\left(\right.$ predicted, $\left.\sigma=1.1 \sigma_{0}\right)$ & $0.154(22 \%)$ & $0.062(6 \%)$ & $0.024(12 \%)$ & $0.015(20 \%)$ & 0.045 \\
\hline$\sqrt{\left\langle a_{n}^{2}\right\rangle}\left(\right.$ predicted, $\left.\sigma=1.2 \sigma_{0}\right)$ & $0.154(22 \%)$ & $0.061(5 \%)$ & $0.021 \quad(9 \%)$ & $0.016(27 \%)$ & 0.047 \\
\hline$\sqrt{\left\langle a_{n}^{2}\right\rangle}\left(\right.$ no-radiation, $\left.\sigma=\sigma_{0}\right)$ & 0.127 & 0.057 & 0.019 & 0.013 & 0.040 \\
\hline
\end{tabular}

TABle 5. Amplitudes of the POD coefficients in the different models. The percentages for the predicted model are expressed relatively to the no-radiation model.

predicted by the observed model $(+30 \%$ compared to the no-radiation model $)$ as well as by the predicted model $(+15 \%)$. The time-averaged energy of the first seven POD modes increases by $37 \%$ in the DNS due to radiative coupling. It increases by $42 \%$ in the observed model and by $37 \%$ in the predicted model compared with the no-radiation model. However, individual amplitudes in the predicted model are not as close to the coupled DNS values as those of the observed model. We note that for all models, the amplitudes of the roll modes $a_{2 / 3}$ tend to decrease as the noise level increases, while those of the boundary layer modes 5 and 6 tend to increase.

Figure 10 compares the symmetrized phase portraits of the DNS and the models in the $\left(a_{2}, a_{3}\right)$ space. Despite the strong similarity, some differences can be noticed between the uncoupled and coupled simulations, as the system spends more time away from the equilibria in the coupled case. This trend is reproduced by the models, as the size of the high probability regions is larger in the observed and predicted models than in the no-radiation model. Once again, the phase portraits of the observed model are in closer agreement with the DNS than those of the predicted model. Figure 11 compares the histogram of the POD amplitude of the $5^{\text {th }}$ mode in the models. We note that the observed model displays the wider distribution which was observed in the DNS (see figure 7), while the predicted model is not able to reproduce this behaviour.

\subsection{Time scales}

Measures of the average time between reorientations $T_{n}$, defined as the mean times between zeros of $a_{n}$ after application of a moving-average filter of $5 T_{c}$, are reported in table 6 . The models reproduce the order of magnitude of $T_{n}$ observed in the simulations and the moderate increase in the reorientation rate when compared with the no-radiation model. Although the values of $T_{n}$ reported in the table correspond to one particular 

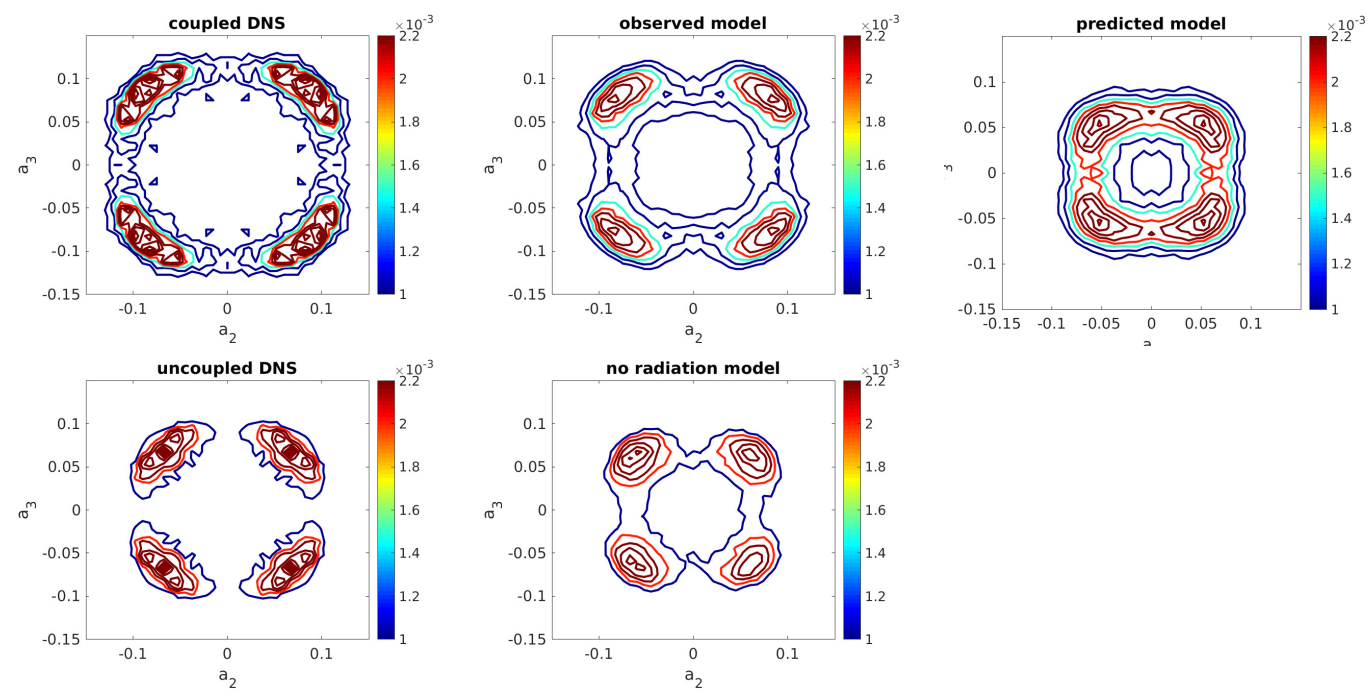

Figure 10. Symmetrized phase portraits of the system; top row, from left to right: coupled DNS, observed model $\left(\sigma=1.2 \sigma_{0}\right)$, predicted model $\left(\sigma=1.2 \sigma_{0}\right)$; bottom row, from left to right: uncoupled DNS, no-radiation model $\left(\sigma=\sigma_{0}\right)$.
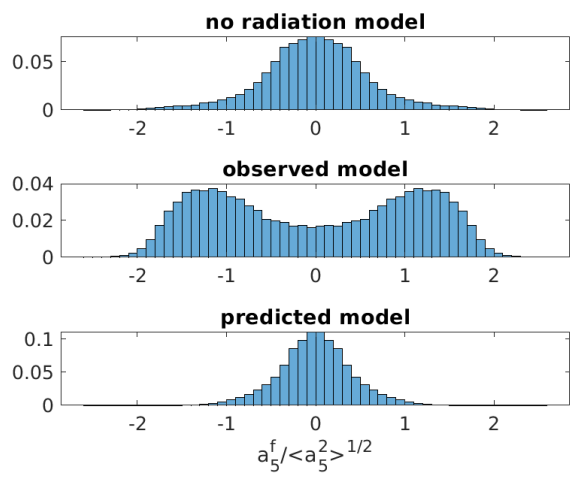

FiguRE 11. Histogram of the filtered model amplitude $a_{5}^{f}$ : no-radiation model (top, $\sigma=\sigma_{0}$ ), predicted model (middle, $\sigma=1.2 \sigma_{0}$ ), observed model (bottom, $\sigma=1.2 \sigma_{0}$ ). A moving average of 100 time units has been applied.

integration of the models, we checked that the order of magnitude did not change over several integrations of the models with different noise signals.

Figure 12 compares the cumulated spectrum of the fluctuating energy of the first seven POD modes for the different models and the DNS. These fluctuations represent $9-10 \%$ of the mean value for all models and simulations with and without radiative coupling. We can see that the effect of radiation in the DNS is to increase the energetic content across the spectrum, for low and high frequencies. The fluctuations also increase over a wide range of frequencies in both models. However, the increase is less marked at low frequencies, in particular for the predicted model, for which no increase is detected below the frequency $f \sim 0.002$. A $15 \%$ increase (compared with $50 \%$ in the simulation) is noted for the models at frequencies below $f_{c} / 2=0.011$, where $T_{c}=1 / f_{c}$ corresponds to the recirculation time associated with fast oscillations of the large-scale circulation. In the range centred around this frequency $\left[f_{c} / 2,2 f_{c}\right]$, the increase is respectively $23 \%$ in the 


$\begin{array}{lccc}\text { Mode } n & 2 & 3 & 4 \\ T_{n} \text { (uncoupled DNS) } & 1258 & 1516 & 703 \\ T_{n} \text { (coupled DNS) } & 1061 & 1412 & 586 \\ \left.T_{n} \text { (no-radiation, } \sigma=\sigma_{0}\right) & 1330 & 1400 & 860 \\ \left.T_{n} \text { (observed, } \sigma=1.2 \sigma_{0}\right) & 1455 & 1535 & 610 \\ \left.T_{n} \text { (observed, } \sigma=1.3 \sigma_{0}\right) & 1090 & 1255 & 585 \\ \left.T_{n} \text { (predicted, } \sigma=1.1 \sigma_{0}\right) & 1300 & 1354 & 740 \\ \left.T_{n} \text { (predicted, } \sigma=1.2 \sigma_{0}\right) & 993 & 1064 & 712\end{array}$

TABLE 6 . Inter-switch periods observed in the simulation and predicted by the different models. The inter-switch period $T_{n}$ is defined as the mean average time between two zeros of $a_{n}$ provided that the time is larger than $5 T_{c}$ where $T_{c}$ corresponds to the high frequency in the simulation (note that this is a slightly different definition from our previous study (Soucasse et al. 2019)). Values given for the models are rounded off to each 5 units.
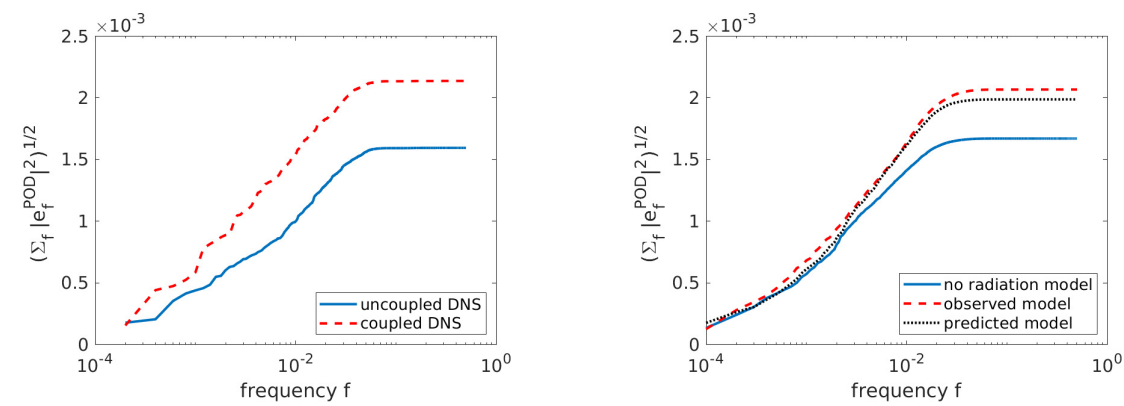

Figure 12. Cumulated spectra of the fluctuations of the POD energy $e^{\mathrm{POD}}(t)=\sum_{n=1}^{7} a_{n}(t)^{2}$ in the DNS (left) and in the models (right). The noise level is $\sigma=\sigma_{0}$ in the no-radiation model and $\sigma=1.2 \sigma_{0}$ in the observed and predicted models.

$\begin{array}{lcccc}n & 1,2 & 3 & 4,5 & 6 \\ \omega_{n} \text { (no-radiation) } & -0.009 \pm 0.0543 i & -0.088 & -0.137 \pm 0.195 i & -0.045 \\ \omega_{n} \text { (observed model) } & -0.010 \pm 0.0585 i & -0.084 & -0.154 \pm 0.208 i & -0.054 \\ \omega_{n} \text { (predicted model) } & -0.007 \pm 0.0591 i & -0.085 & -0.136 \pm 0.209 i & -0.049\end{array}$

TABLE 7. Eigenvalues of the linearised model around the equilibria.

DNS, while it is about $30 \%$ for the predicted model and $40 \%$ for the observed model. Over the full frequency range, the increase in the fluctuations is $34 \%$ in the DNS, while it is $19 \%$ for the predicted model and $24 \%$ for the observed model (see figure 12).

Further insight into the dynamics of the model can be provided by linear stability analysis around the equilibria. As seen in Soucasse et al. (2019), the equilibria are stable but can be destabilized in the presence of noise due to the existence of a weakly stable direction, corresponding to the least stable pair of eigenmodes. In particular, a connection can be established between high frequencies in the model and the imaginary part of the least stable pair of eigenvalues. Table 7 shows that in the observed and predicted models the least stable pair of eigenvalues has an imaginary part of 0.0585 and 0.0591 respectively, compared with 0.0543 for the case without radiation, which corresponds to relative increases of $8 \%$ and $9 \%$ respectively, to be compared with the value of $13 \%$ observed in the simulation.

To sum up, the observed model is able to reproduce energy levels, higher LSC reorien- 
tation rates, faster oscillation times, and qualitative changes in the statistics of the POD amplitudes due to radiative coupling. The predicted model, which is exclusively based on uncoupled data, is not as accurate as the observed model but is still able to predict an increase in the energy of the modes and a higher LSC reorientation rate.

\section{Conclusion}

DNS of coupled natural convection and thermal radiation has been performed in a cubic Rayleigh Bénard cell at $R a=10^{7}$ for an air $/ \mathrm{H}_{2} \mathrm{O} / \mathrm{CO}_{2}$ mixture at room temperature. Results show that radiation strengthens the convection, increases the kinetic energy of the flow and, to a lesser extent, increases the temperature fluctuations. In addition, radiation alters the reorientation dynamics of the LSC: the reorientations seem to be more frequent and the flow spends more time outside the four quasi-stable flow states. A POD analysis reveals that compared to the uncoupled case, the first POD eigenfunctions are preserved while POD eigenvalues are increased when radiation is considered.

In order to capture these effects, a POD-based reduced-order modelling strategy has been proposed. The approach extends standard POD modelling of Navier-Stokes equation by the addition of a radiation term in the model. This radiation term is obtained from a rigorous projection of the radiative power onto the POD basis set. The linearisation of radiation emission (consistent with Boussinesq approximation) is used to decompose the radiative power into a sum of modal contributions. From this approach, two models have been considered. The first one is based on eigenfunctions and eigenvalues computed from the radiatively coupled DNS. This observed model is able to reproduce the change in dynamics associated with the coupling, in particular higher reorientation rates and faster oscillations for the LSC. The second model is derived from POD results obtained for the uncoupled case. This predicted model is able to foresee some of the increase in the energy levels due to the effect of radiation. It also predicts a higher reorientation rate, in good agreement with the observations made from the simulation. The study shows that the POD-based reduced-order modelling approach is not only able to reproduce the changes in dynamics due to moderate radiative coupling, but that it can also predict them, at least in part.

To the best of our knowledge, this is the first time a POD-based low order model for natural convection is derived taking into account radiation effects. Although we have studied a particular configuration (given Rayleigh number, given radiating species concentration, etc.), the proposed methodology is applicable to any coupled natural convection-radiation problem under Boussinesq approximation. The validity and accuracy of the predicted approach of radiation effects from uncoupled data is not guaranteed for large changes in radiation parameters. However, this predicted approach can be used as an exploratory tool, to investigate the influence of radiative transfer at low computational cost.

\section{Acknowledgements}

This work was granted access to the HPC resources of IDRIS under the allocation 2019A0042B00209 attributed by GENCI (Grand Equipement National de Calcul Intensif).

\section{Declaration of Interests}

The authors report no conflict of interest. 
Reduced-order modelling of radiative transfer effects on Rayleigh-Bénard convection 23

\section{Appendix A. Derivation of the modal-radiative power}

This appendix details the derivation of the modal-radiative power $\mathcal{P}_{n}^{\text {rad }}$ introduced in section 4.1.1. Owing to the weak temperature gradients, the power fourth temperature field can be linearised around the mean temperature $T_{0}$ and the POD decomposition of the temperature field can be introduced

$$
T^{4}(\boldsymbol{r}, t) \simeq T_{0}^{4}+4 T_{0}^{3} \Delta T \sum_{n} a_{n}(t) \phi_{n}^{\theta}(\boldsymbol{r}) .
$$

Because of the linearity of the radiative transfer equation (equation (2.7)), the radiative intensity field $I_{j}(\boldsymbol{\Omega}, \boldsymbol{r})$ can be decomposed similarly

$$
I_{j}(\boldsymbol{\Omega}, \boldsymbol{r}, t)=\frac{w_{j} \sigma}{\pi} T_{0}^{4}+\frac{w_{j} \sigma 4 T_{0}^{3} \Delta T}{\pi} \sum_{n} a_{n}(t) \psi_{j, n}^{\theta}(\boldsymbol{\Omega}, \boldsymbol{r}),
$$

where $\psi_{j, n}^{\theta}(\boldsymbol{\Omega}, \boldsymbol{r})$ is a modal-intensity field for the $j^{\text {th }} k$-class, associated with POD mode temperature field $\phi_{n}^{\theta}(\boldsymbol{r})$ and satisfying the following transport equation

$$
\boldsymbol{\Omega} \cdot \boldsymbol{\nabla} \psi_{j, n}^{\theta}(\boldsymbol{\Omega}, \boldsymbol{r})=k_{j}\left(\phi_{n}^{\theta}(\boldsymbol{r})-\psi_{j, n}^{\theta}(\boldsymbol{\Omega}, \boldsymbol{r})\right),
$$

and boundary condition for $\boldsymbol{\Omega} \cdot \boldsymbol{n}>0$

$$
\left.\psi_{j, n}^{\theta}\left(\boldsymbol{\Omega}, \boldsymbol{r}^{w}\right)=\varepsilon \phi_{n}^{\theta}\left(\boldsymbol{r}^{w}\right)\right)+\frac{1-\varepsilon}{\pi} \int_{\boldsymbol{\Omega}^{\prime} \cdot \boldsymbol{n}<0} \psi_{j, n}^{\theta}\left(\boldsymbol{\Omega}^{\prime}, \boldsymbol{r}^{w}\right)\left|\boldsymbol{\Omega}^{\prime} \cdot \boldsymbol{n}\right| d \boldsymbol{\Omega}^{\prime} .
$$

Once equations (A 3)-(A 4$)$ are solved for each $k$-class using the ray-tracing algorithm detailed in section 2.2 , one can compute a modal-radiative power associated with the $n^{\text {th }}$ POD mode

$$
\mathcal{P}_{n}^{\mathrm{rad}}(\boldsymbol{r})=\frac{L^{2}}{\lambda} \frac{\sigma 4 T_{0}^{3}}{\pi} \sum_{j} k_{j} w_{j}\left(\int_{4 \pi} \psi_{j, n}^{\theta}(\boldsymbol{\Omega}, \boldsymbol{r}) d \boldsymbol{\Omega}-4 \pi \phi_{n}^{\theta}(\boldsymbol{r})\right),
$$

and the radiation matrix, as defined in equation (4.3). The total radiative power is then the sum of all modal-radiative powers, weighted by the associated POD coefficient (equation (4.2)).

\section{Appendix B. Model parameters}

This appendix provides model parameters $L^{D}, L^{B}, L^{R}$ and $Q$, defined by

$$
\begin{gathered}
L_{n m}^{B}=\int \operatorname{Pr} \phi_{\theta}^{m} \phi_{3}^{n} d \boldsymbol{r} \\
L_{n m}^{D}=\int\left[\frac{P r}{\sqrt{R a}} \frac{\partial^{2} \phi_{i}^{m}}{\partial x_{j} \partial x_{j}} \phi_{i}^{n}+\frac{\gamma^{2}}{\sqrt{R a}} \frac{\partial^{2} \phi_{\theta}^{m}}{\partial x_{j} \partial x_{j}} \phi_{\theta}^{n}\right] d \boldsymbol{r}, \\
L_{n m}^{R}=\int \frac{\gamma^{2}}{\sqrt{R a}} \mathcal{P}_{m}^{\mathrm{rad}}(\boldsymbol{r}) \phi_{n}^{\theta}(\boldsymbol{r}) d \boldsymbol{r}, \\
Q_{n m p}=\int\left[-\phi_{j}^{p} \frac{\partial \phi_{i}^{m}}{\partial x_{j}} \phi_{i}^{n}-\gamma^{2} \phi_{j}^{p} \frac{\partial \phi_{\theta}^{m}}{\partial x_{j}} \phi_{\theta}^{n}\right] d \boldsymbol{r} .
\end{gathered}
$$

$L^{B}, L^{D}$ and $L^{R}$ are linear contributions in the model arising from Galerkin projection of buoyancy term, diffusion terms and radiative term in equations (2.2)-(2.3) onto the POD basis. Similarly, $Q$ is a quadratic contribution in the model arising from Galerkin 


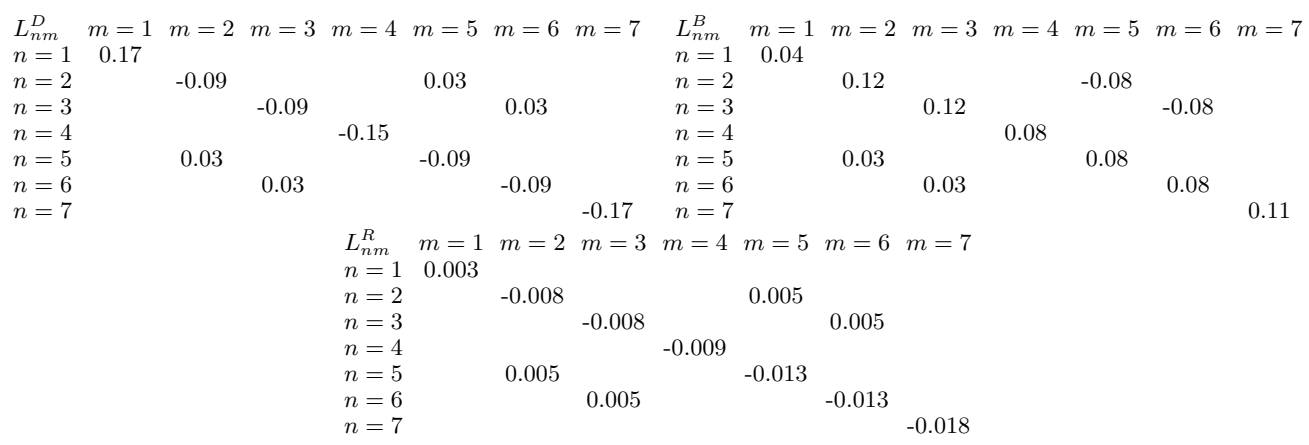

TABle 8. Linear diffusion matrix (top left), linear buoyancy matrix (top right) and linear radiation matrix (bottom) computed from coupled POD eigenfunctions. Entries below $10^{-5}$ are left empty.

projection of advection terms in equations (2.2)-(2.3) onto the POD basis. More details on the derivation of these terms can be found for instance in Podvin \& Le Quéré (2001).

In the observed model, these quantities are determined from coupled POD eigenfunctions, and values are reported in tables 8 and 9 . In the no-radiation model and in the predicted model, these quantities are determined from uncoupled eigenfunctions; values of parameters $L^{D}, L^{B}$ and $Q$ can be found in Soucasse et al. (2019) and values of parameter $L^{R}$ (for the predicted model only) are given in table 10. Uncoupled POD eigenfunction and coupled POD eigenfunctions being very similar, differences in parameter values are mostly due to differences in the value of $\gamma$ that increases when coupling with radiation.

\section{REFERENCES}

BAI, K., Ji, D. \& Brown, E. 2016 Ability of a low-dimensional model to predict geometrydependent dynamics of large-scale coherent structures in turbulence. Physical Review E 93, 023117 .

Bailon-Cuba, J., Emran, M. S. \& Schumacher, J. 2010 Aspect ratio dependece of heat transfer and large-scale flow in turbulent convection. Journal of Fluid Mechanics 655, $152-173$.

BdÉoui, F. \& Soufiani, A. 1997 The onset of Rayleigh-Bénard instability in molecular radiating gases. Physics of Fluids 9, 3858-3872.

Berkooz, G., Holmes, P. \& Lumley, J. L. 1993 The proper orthogonal decomposition in the analysis of turbulent flows. Annual Review of Fluid Mechanics 25, 539-575.

Borget, V., BdÉoui, F., Soufiani, A. \& Le Quéré, P. 2001 The transverse instability in a differentially heated vertical cavity filled with molecular radiating gases. I. Linear stability analysis. Physics of Fluids 13 (5), 1492-1507.

Boulllaut, V., Lepot, S., Aumaître, S. \& Gallet, B. 2019 Transition to the ultimate regime in a radiatively driven convection experiment. Journal of Fluid Mechanics 861 (R5).

Brown, E., Nikolaenko, A. \& Ahlers, G. 2005 Reorientation of the large-scale circulation in turbulent Rayleigh-Bénard convection. Physical Review Letters 95, 084503.

Czarnota, T. \& Wagner, C. 2016 Turbulent convection and thermal radiation in a cuboidal Rayleigh-Bénard cell with conductive plates. International Journal of Heat and Fluid Flow 57, 150-172.

Foroozani, N., Niemela, J. J., Armenio, V. \& Sreenivasan, K. R. 2017 Reorientations of the large-scale flow in turbulent convection in a cube. Physical Review E 95, 033107. 


$$
\begin{aligned}
& Q_{1 m p} \quad p=1 \quad p=2 p=3 \quad p=4 \quad p=5 \quad p=6 \quad p=7 \\
& m=1 \\
& m=2 \\
& m=3 \\
& m=4 \\
& m=5 \\
& m=6 \\
& m=7 \\
& -0.58 \\
& \begin{array}{llll} 
& -0.58 & & -0.05 \\
-0.05 & & -0.87 & \\
& -0.05 & & -0.74
\end{array} \\
& -0.05 \\
& -0.74 \\
& Q_{7 m p} \quad p=1 \quad p=2 p=3 \quad p=4 \quad p=5 \quad p=6 \quad p=7 \\
& m=1 \quad 0.54 \\
& \begin{array}{lllll}
m=2 & -0.61 & & -0.12 & \\
m=3 & & 0.61 & & 0.12
\end{array} \\
& m=4 \\
& \begin{array}{lllll}
m=5 & -0.12 & & -0.48 & \\
m=6 & & 0.12 & & 0.48
\end{array} \\
& \begin{array}{ll}
m=7 & 0.54
\end{array}
\end{aligned}
$$
$-1.76$
0.24

0.07

.71

TABLE 9. Quadratic interaction matrices computed from coupled POD eigenfunctions (in symmetric form i.e. corresponding to $Q_{n m p}+Q_{n p m}$ for $p \neq m$ and $Q_{n m p}$ for $p=m$ ). Entries below $10^{-5}$ are left empty.

$$
\begin{array}{lccccccc}
L_{n m}^{R} & m=1 & m=2 & m=3 & m=4 & m=5 & m=6 & m=7 \\
n=1 & 0.002 & & & & & & \\
n=2 & & -0.008 & & & 0.004 & & \\
n=3 & & & -0.008 & & & 0.004 & \\
n=4 & & & & -0.007 & & & \\
n=5 & & 0.004 & & & -0.011 & & \\
n=5 & & & 0.004 & & & -0.011 & \\
n=6 & & & & & & & -0.014 \\
n=7 & & & & & & &
\end{array}
$$

TABLE 10. Linear radiation matrix computed from uncoupled POD eigenfunctions. Entries below $10^{-5}$ are left empty.

Gille, J. \& Goody, R. 1964 Convection in a radiating gas. Journal of Fluid Mechanics 20, $47-79$.

Goody, R. M. 1956 The influence of radiative transfer on cellular convection. Journal of Fluid Mechanics 1, 424-435.

Grossmann, S. \& Lohse, D. 2000 Scaling in thermal convection: a unifying theory. Journal of Fluid Mechanics 407, 2756.

Holmes, P., Lumley, J. \& Berkooz, G. 1996 Turbulence, Coherent Structures, Dynamical Systems and Symmetry. Cambridge University Press. 
Hutchinson, J. E. \& Richards, R. F. 1999 Effect of nongray gas radiation on thermal stability in carbon dioxied. Journal of Thermophysics and Heat Transfer 13, 25-32.

Kogawa, T., Okajima, J., Sakurai, A., Komiya, A. \& Maruyama, S. 2017 Influence of radiation effect on turbulent natural convection in cubic cavity at normal temperature atmospheric gas. International Journal of Heat and Mass Transfer 104, 456466.

Lan, C. H., Ezekoye, O. A., Howell, J. R. \& Ball, K. S. 2003 Stability analysis for threedimensional Rayleigh-Bénard convection with radiatively participating medium using spectral methods. International Journal of Heat and Mass Transfer 46, 1371-1383.

Lepot, S., Aumaître, S. \& Gallet, B. 2018 Radiative heating achieves the ultimate regime of thermal convection. Proceedings of the National Academy of Sciences 115 (36).

Lohse, Detlef \& XiA, Ke-Qing 2010 Small-scale properties of turbulent Rayleigh-Bénard convection. Annual Review of Fluid Mechanics 42 (1), 335-364.

Lumley, J.L. \& Podvin, B. 1996 Dynamical systems theory and extra rates of strain in turbulent flows. Journal of Experimental and Thermal Fluid Science 267, 1-10.

Mishra, P. K., De, A. K., Verma, M. K. \& Eswaran, V. 2011 Dynamics of reorientations and reversals of large-scale flow in Rayleigh-Bénard convection. Journal of Fluid Mechanics 668, 480-499.

Mishra, S. C., Akhtar, A. \& Garg, A. 2014 Numerical analysis of Rayleigh-Bénard convection with and without volumetric radiation. Numerical Heat Transfer, Part A: Applications 65 (2), 144-164.

Park, H. M., Sung, M.C. \& Chung, J. S. 2004 Stabilization of Rayleigh-Bénard convection by means of mode reduction. Proceedings of the Royal Society A 460, 1807-1830.

Pierrot, L., Rivière, P., Soufiani, A. \& Taine, J. 1999 A fictitious-gas-based absorption distribution function global model for radiative transfer in hot gases. Journal of Quantitative Spectroscopy and Radiative Transfer 62, 609-624.

Podvin, B. \& Le QuÉRÉ, P. 2001 Low-order models for the flow in a differentially heated cavity. Physics of Fluids 13 (11), 3204-3214.

Podvin, B. \& Sergent, A. 2015 A large-scale investigation of wind reversal in a square Rayleigh-Bénard cell. Journal of Fluid Mechanics 766, 172-201.

Podvin, B. \& Sergent, A. 2017 Precursor for wind reversal in a square Rayleigh-Bénard cell. Physical Review E 95 (013112).

Prasanna, Swaminathan \& Venkateshan, S. P. 2014 Convection induced by radiative cooling of a layer of participating medium. Physics of Fluids 26 (5), 056603.

Puiguaner, D., Herrero, J., Simo, C. \& Giralt, F. 2008 Bifurcation analysis of steady Rayleigh-Bénard convection in a cubical cavity with conducting sidewalls. Journal of Fluid Mechanics 598, 393-427.

Sakurai, A., Matsubara, K., Takakuwa, K. \& Kanbayashi, R. 2012 Radiation effects on mixed turbulent natural and forced convection in a horizontal channel using direct numerical simulation. International Journal of Heat and Mass Transfer 55, 2539-2548.

Shishkina, O., Stevens, R. J. A. M., Grossmann, S. \& Lohse, D. 2010 Boundary layer structure in turbulent thermal convection and its consequences for the required numerical resolution. New Journal of Physics 12, 075022.

Sirovich, L. 1987 Turbulence and the dynamic of coherent structures. Part I: Coherent structures. Quarterly of Applied Mathematics 45 (3), 561-571.

SoucAsse, L. 2013 Effets des transferts radiatifs sur les écoulements de convection naturelle dans une cavité différentiellement chauffée en régimes transitionnel et faiblement turbulent. $\mathrm{PhD}$ thesis, École Centrale Paris, France.

Souchsse, L., Podvin, B., Rivière, Ph. \& Soufiani, A. 2019 Proper orthogonal decomposition analysis and modelling of large-scale flow reorientations in a cubic RayleighBénard cell. Journal of Fluid Mechanics 881, 23-50.

Souchsse, L., Rivière, Ph. \& Soufiani, A. $2014 a$ Effects of molecular gas radiation on Rayleigh-Bénard convection in a 3D cubical cavity. In Proceedings of the 15th International Heat Transfer Conference, pp. IHTC15-9563.

Soucasse, L., Rivière, Ph. \& Soufiani, A. 2016 Natural convection in a differentially heated cubical cavity under the effects of wall and molecular gas radiation at Rayleigh numbers up to $3 \times 10^{9}$. International Journal of Heat and Fluid Flow 61-B, 510-530.

Souchsse, L., Rivière, Ph., Soufiani, A., Xin, S. \& Le Quéré, P. $2014 b$ Transitional 
regimes of natural convection in a differentially heated cavity under the effects of wall and molecular gas radiation. Physics of Fluids 26, 024105.

Soucasse, L., Rivière, Ph., Xin, S., Le Quéré, P. \& Soufiani, A. 2012 Numerical study of coupled molecular gas radiation and natural convection in a differentially heated cubical cavity. Computational Thermal Sciences 4, 335-350.

Soufiani, A. 1991 Temperature turbulence spectrum for high-temperature radiating gases. Journal of Thermophysics 5 (4), 489-494.

SpIEgel, E. A. 1960 The convective instability of a radiating fluid layer. Astrophysical Journal 132, 716-728.

van der Poel, E. P., Verzicco, R., Grossmann, S. \& Lohse, D. 2015 Plume emission statistics in turbulent Rayleigh-Bénard convection. Journal of Fluid Mechanics 772, 515.

Vasiliev, A., Frick, P., Kumar, A., Stepanov, R., Sukhanovskit, A. \& Verma, M. K. 2019 Transient flows and reorientations of large-scale convection in a cubic cell. International Communications in Heat and Mass Transfer 108, 104319.

Verdoold, J., Tummers, M. J. \& Hanjalić, K. 2009 Prime modes of fluid circulation in large-aspect-ratio turbulent Rayleigh-Bénard convection. Physical Review E 80, 037301.

Xin, S., Chergui, J. \& Le Quéré, P. 2008 3D spectral parallel multi-domain computing for natural convection flows. In Parallel Computational Fluid Dynamics, pp. 163-171.

XIN, S. \& LE Quéré, P. 2002 An extended Chebyshev pseudo-spectral benchmark for the 8:1 differentially heated cavity. Int. J. Heat Mass Transfer 40, 981-998. 